\title{
A FINITE ELEMENT HETEROGENEOUS MULTISCALE METHOD WITH IMPROVED CONTROL OVER THE MODELING ERROR*
}

\author{
DOGHONAY ARJMAND ${ }^{\dagger}$ AND CHRISTIAN STOHRER ${ }^{\ddagger}$
}

\begin{abstract}
Multiscale partial differential equations (PDEs) are difficult to solve by traditional numerical methods due to the need to resolve the small wavelengths in the media over the entire computational domain. We develop and analyze a Finite Element Heterogeneous Multiscale Method (FE-HMM) for approximating the homogenized solutions of multiscale PDEs of elliptic, parabolic, and hyperbolic type. Typical multiscale methods require a coupling between a micro and a macro model. Inspired from the homogenization theory, traditional FE-HMM schemes use elliptic PDEs as the micro model. We use, however, the second order wave equation as our micro model independent of the type of the problem on the macro level. This allows us to control the modeling error originating from the coupling between the different scales. In a spatially fully discrete a priori error analysis we prove that the modeling error can be made arbitrarily small for periodic media, even if we do not know the exact period of the oscillations in the media. We provide numerical examples in one and two dimensions confirming the theoretical results. Further examples show that the method captures the effective solutions in general non-periodic settings as well.
\end{abstract}

Key words. Multiscale method, homogenization, partial differential equations, modeling error.

AMS subject classifications. 35B27, 35J15, 65M60, 65N30, 74Q10, 74Q15.

\section{Introduction}

Various physical phenomena in the universe have multiscale/multiphysics nature. In general, attacking these kind of problems poses a considerable difficulty due to the interaction of different scales in the problem. While the smallest scales in the problem contribute to the average behavior of the overall system, the quantity of interest - the average part of the multiscale solution - varies on a larger scale only. Nevertheless, using a coarse resolution in a naive way leads to significant errors. On the other hand, resolving all the scales in the problem through the entire computational domain can be prohibitively expensive if the size of the fluctuations in the model parameters are much smaller than the characteristic length of the domain. Therefore, the trend has been to develop multiscale methods which couple the mathematical models in different scales in a clever way and thereby lead to a lower computational cost, see [14] and the references therein.

In this work, we develop and analyze a multiscale method, within the framework of Heterogeneous Multiscale Methods (HMM). This framework was introduced by E and Engquist [15] to treat problems with multiscale nature. In the last decade multiscale schemes based on HMM principles have been successfully applied to a variety of different multiscale problems $[3,16]$. Here, we propose a novel HMM scheme, which approximates the effective solutions of multiscale problems modeled by linear elliptic, parabolic, and hyperbolic partial differential equations (PDEs). We assume that the multiscale nature of the problem is only due to the heterogeneities in the medium, e.g., in the elliptic case

*Received: July 23, 2014; accepted: April 1, 2015. Communicated by Weinan E.

${ }^{\dagger}$ Department of Mathematics and Swedish e-Science Research Center (SeRc), Royal Institute of Technology (KTH), Sweden (doghonay@kth.se). http://www.nada.kth.se/ ${ }^{\text {doghonay/ }}$

${ }^{\ddagger}$ Laboratoire POEMS, UMA, ENSTA ParisTech, 828, Boulevard des Marchaux, 91762 Palaiseau Cedex, France (christian.stohrer@ensta-paristech.fr). http://uma.ensta-paristech.fr/ ${ }^{\text {stohrer. }}$ 
we consider the model problem

$$
\left\{\begin{array}{l}
\text { Find } u^{\varepsilon} \in H_{0}^{1}(\Omega), \text { such that } \\
\int_{\Omega} a^{\varepsilon}(x) \nabla u^{\varepsilon} \cdot \nabla v d x=\int F v d x \quad \forall v \in H_{0}^{1}(\Omega) .
\end{array}\right.
$$

Here the properties of the medium is represented by the coefficient $a^{\varepsilon}$, where $\varepsilon$ is the length of its fastest oscillations and thus stands for the size of the smallest scale in the problem. We will introduce the precise mathematical settings of our model problems in the next section. As $\varepsilon$ gets smaller, a direct numerical simulation of such a problem becomes expensive. On the contrary, the solution $u^{\varepsilon}$ converges to the solution of an effective problem, known as the homogenized problem, which has no dependency on the small scale parameter. This is a well known result from homogenization theory (see $[10,13,21])$. Nevertheless, explicit formulas for the homogenized equation are only available under certain restrictive assumptions such as periodicity of the media. Our motivation in this paper is to develop a method with targeting three main goals. First, the method should not assume any restrictive assumptions such as periodicity. The only assumption, other than classical well-posedness requirements, is that the scales involved in the problem should be well separated. Second, the method should approximate the homogenized solution up to high orders whenever homogenization is applicable, e.g. in periodic media. Lastly, the method should be far cheaper than the traditional methods.

The principal idea of HMM is to solve an incomplete macro problem with a standard numerical scheme. The missing data to solve the problem are computed on the fly by solving micro problems constrained by the macro state. Since the micro problems are computed on small sampling domains not covering the entire computational domain HMM schemes become feasible in terms of computational costs. Moreover, since the micro problems are independent of each other they could be solved in parallel reducing the computational time even further. Following this idea one has to choose two solvers, one for the solution of the macro problem and one for the micro problem. Using twice a finite element method is a common choice, leading to so called FE-HMM schemes.

The FE-HMM scheme proposed in $[1,17]$ for the elliptic, in $[6,23]$ for the parabolic, and in $[4,5]$ for the hyperbolic problem, use elliptic micro problems to compute the FEHMM bilinear form. At first, it might be surprising, that the same micro problem is used in all three cases. Homogenization theory gives a profound explanation thereof. Since only the elliptic part of all three second order partial differential equations displays a multiscale behavior, the homogenized tensor remains the same for all three cases. The a priori error analysis of these FE-HMM schemes relies on Strang-type lemmas for nonconforming FEMs and the decomposition of the overall error into a macro, a micro, and a modeling error. The former two errors can be controlled by choosing appropriate meshsizes. However, in a general non-periodic setting, or if the true period of a material is unknown a non-negligible modeling error arises mainly caused by the artificial boundary conditions for the micro problems [26].

On the contrary, by the physical intuition it is natural to have the same type of problems in the macro and the micro levels. For instance, Engquist, Holst, and Runborg proposed in $[18,19]$ a FD-HMM scheme for the wave equation with a hyperbolic micro problem, instead of the elliptic one. Later the idea of using the wave equation as the micro model was applied to FD-HMM scheme for elliptic problems [7]. The motivation behind this strategy is to remove the boundary error which originates from the artificial boundary condition posed on the micro problem. Due to the finite speed of propagation of waves, the error on the boundary does not influence the interior solution if the 
micro domain is large enough. However, when formulated in a finite element setting, this method does not lead immediately to a symmetric bilinear form, which makes the complete convergence analysis hard to carry through [25, Section 5.5].

Inspired by this FD-HMM scheme, we propose and analyze here a FE-HMM, which uses hyperbolic micro problems. Therefore, it does not suffer from the artificial boundary error in the micro problem due to the finite speed of propagation of the microscopic solutions. Moreover, the method leads to a symmetric bilinear form. Hence, it is amenable to a rigorous mathematical study. Similar as for the standard FE-HMM our new scheme can be used to approximate the homogenized solutions of elliptic, parabolic, and hyperbolic equations, without needing to precompute the homogenized coefficients, nor to resolve the microscopic scale underlying media. In addition, the method does not assume any precise knowledge about the wavelength of the fluctuations in the media. To validate the reliability of our method, we consider a rather academic case of purely periodic media and we prove that the solution of the proposed method converges to the homogenized solution up to arbitrary order in terms of $\varepsilon / \eta$, where $\eta$ is the size of the micro domain. Furthermore, we provide numerical results to support the theoretical arguments.

The discussion in this paper is organized as follows. In Section 2 we introduce the precise mathematical settings we are interested in. The multiscale method is presented in Section 3. Section 4 contains our main statements. The main result is Theorem 4.7 in which we prove that our method improves the modeling error up to arbitrary rates in $\varepsilon / \eta$. Moreover, we use this theorem to estimate the difference between the FE-HMM and the homogenized solutions. The last section is devoted to the numerical study of the method. We consider problems in one and two dimensions showing that the effective parameters are well-captured.

\section{Model problems}

Let $\Omega$ be a bounded Lipschitz domain in $\mathbb{R}^{d}, 1 \leq d \leq 3$. For simplicity we assume that $\Omega$ can be covered exactly with simplicial or quadrilateral elements. We consider three classical model problems for second order partial differential equation in their variational form and equipped with homogeneous Dirichlet boundary conditions. Note that the choice for this type of boundary condition is only for the ease of presentation. Yet, other boundary conditions could be used as well with no conceptual changes in the multiscale method. Hence, we consider the elliptic problem

$$
\left\{\begin{array}{l}
\text { Find } u^{\varepsilon} \in H_{0}^{1}(\Omega), \text { such that } \\
B^{\varepsilon}\left(u^{\varepsilon}, v\right)=(F, v) \quad \forall v \in H_{0}^{1}(\Omega),
\end{array}\right.
$$

the heat equation

$$
\left\{\begin{aligned}
\text { Find } u^{\varepsilon}:[0, T] \rightarrow H_{0}^{1}(\Omega), \text { such that } & & \\
\left(\partial_{t} u^{\varepsilon}(t), v\right)+B^{\varepsilon}\left(u^{\varepsilon}(t), v\right)=(F(t), v) & & \forall v \in H_{0}^{1}(\Omega), 0<t<T, \\
u^{\varepsilon}(0)=f & & \text { in } \Omega,
\end{aligned}\right.
$$

and the wave equation

$$
\left\{\begin{aligned}
\text { Find } u^{\varepsilon}:[0, T] \rightarrow H_{0}^{1}(\Omega), & \text { such that } & & \\
\left(\partial_{t t} u^{\varepsilon}(t), v\right)+B^{\varepsilon}\left(u^{\varepsilon}(t), v\right) & =(F(t), v) & & \forall v \in H_{0}^{1}(\Omega), 0<t<T, \\
u^{\varepsilon}(0) & =f & & \text { in } \Omega, \\
\partial_{t} u^{\varepsilon}(0) & =g & & \text { in } \Omega .
\end{aligned}\right.
$$


Here $(\cdot, \cdot)$ denotes the standard $L^{2}$-inner product over $\Omega$ and the bilinear form $B^{\varepsilon}$ is given by

$$
B^{\varepsilon}(v, w)=\int_{\Omega} a^{\varepsilon}(x) \nabla v(x) \cdot \nabla w(x) d x, \quad \forall v, w \in H_{0}^{1}(\Omega) .
$$

We also assume that $a^{\varepsilon} \in L^{\infty}\left(\Omega ; \mathbb{R}^{d \times d}\right)$ is symmetric, uniformly elliptic and bounded, i.e., there exist $0<\alpha \leq \beta$, such that for all $\xi \in \mathbb{R}^{d}$ and for all $\varepsilon>0$

$$
\alpha|\xi|^{2} \leq a^{\varepsilon}(x) \xi \cdot \xi \leq \beta|\xi|^{2} .
$$

Hence the bilinear form $B^{\varepsilon}$ is symmetric, uniformly elliptic and bounded on $H_{0}^{1}(\Omega)$. Note that the coefficient $a^{\varepsilon}$ is assumed to be time independent and our theoretical results for the parabolic and hyperbolic problems, (2) and (2), do not apply directly to general time dependent coefficients. Furthermore, we make the following standard regularity assumptions:

for the elliptic problem (2): $F \in L^{2}(\Omega)$

for the heat equation (2): $F \in L^{2}\left(0, T ; L^{2}(\Omega)\right), \quad f \in L^{2}(\Omega)$

for the wave equation (2): $F \in L^{2}\left(0, T ; L^{2}(\Omega)\right), \quad f \in H_{0}^{1}(\Omega), \quad g \in L^{2}(\Omega)$.

It is well known, that under these assumptions (2), (2), and (2) have each a unique (weak) solution in the appropriate functional spaces.

2.1. Homogenization theory. By classical results in homogenization theory the solutions of all three model problem converge to a homogenized solution $u^{0}$ as $\varepsilon \rightarrow 0$. These limits solve the so-called homogenized problems, given by

$$
\left\{\begin{array}{l}
\text { Find } u^{0} \in H_{0}^{1}(\Omega), \text { such that } \\
B^{0}\left(u^{0}, v\right)=(F, v) \quad \forall v \in H_{0}^{1}(\Omega),
\end{array}\right.
$$

for the elliptic problem (2),

$$
\left\{\begin{aligned}
\text { Find } u^{0}:[0, T] \rightarrow H_{0}^{1}(\Omega), \text { such that } & \\
\left(\partial_{t} u^{0}(t), v\right)+B^{0}\left(u^{0}(t), v\right)=(F(t), v) & \forall v \in H_{0}^{1}(\Omega), 0<t<T, \\
u^{0}(0)=f & \text { in } \Omega,
\end{aligned}\right.
$$

for the heat equation (2.1), and

$$
\left\{\begin{array}{rlrl}
\text { Find } u^{0}:[0, T] \rightarrow H_{0}^{1}(\Omega), & \text { such that } & & \\
\left(\partial_{t t} u^{0}(t), v\right)+B^{0}\left(u^{0}(t), v\right)=(F(t), v) & & \forall v \in H_{0}^{1}(\Omega), 0<t<T, \\
u^{0}(0)=f & & \text { in } \Omega, \\
\partial_{t} u^{0}(0)=g & & \text { in } \Omega .
\end{array}\right.
$$

for the wave equation (2). In brief summary: The variational formulations of the homogenized equations of all three model are given by replacing the bilinear form $B^{\varepsilon}$ with the homogenized bilinear form $B^{0}$ defined as

$$
B^{0}(v, w)=\int_{\Omega} a^{0}(x) \nabla v(x) \cdot \nabla w(x) d x \quad \forall v, w \in H_{0}^{1}(\Omega) .
$$


Here $a^{0}$ is the standard homogenized matrix, which is also symmetric and satisfies (2). We recall that in the periodic case, i.e. $a^{\varepsilon}(x)=a(x / \varepsilon)$, where $a$ is $Y$-periodic and $Y=[0,1]^{d}$ the $d$-dimensional unit cube, the homogenized matrix is constant and given by

$$
a_{i, j}^{0}=\int_{Y} a(x)\left(e_{j}+\nabla \chi^{j}(x)\right) \cdot e_{i} d x
$$

where $e_{i}$ denotes the $i$-th canonical basis vector of $\mathbb{R}^{d}$ and $\chi^{i}$ solves the cell problem

$$
\int_{Y} a(x) \nabla \chi^{i}(x) \cdot \nabla z(x) d x=-\int_{Y} a(x) e_{i} \cdot \nabla z(x) d x, \quad \forall z(x) \in W_{\mathrm{per}}^{1}(Y) .
$$

The proofs of the above statements and further details about homogenization can be found in, e.g., $[10,13]$.

\section{Multiscale method}

Like standard FE-HMM our scheme is a non-conforming FEM on a coarse (macroscopic) mesh. On the macroscopic level there are no essential changes compared with the former FE-HMM schemes. However, on the microscopic level, the use of a hyperbolic micro problem to estimate the effective bilinear form is a novel approach.

First of all we introduce the notation we will use. Let $\mathcal{T}_{H}$ be a triangulation of $\Omega$ into either simplicial or quadrilateral elements $K$. We assume that all elements $K \in \mathcal{T}_{H}$ are affine equivalent to a reference element $\hat{K}$. The affine mapping from $\hat{K}$ to $K$ is denoted by $F_{K}$. We denote the macro finite element spaces by

$$
S^{\ell}\left(\Omega, \mathcal{T}_{H}\right)=\left\{v_{H} \in H^{1}(\Omega):\left.v_{H}\right|_{K} \in \mathcal{R}^{\ell}(K), \forall K \in \mathcal{T}_{H}\right\}
$$

and $S_{0}^{\ell}\left(\Omega, \mathcal{T}_{H}\right)=S^{\ell}\left(\Omega, \mathcal{T}_{H}\right) \cap H_{0}^{1}(\Omega)$, where $\mathcal{R}^{\ell}(K)=\mathcal{P}^{\ell}(K)$, the space of polynomials of degree $\ell$ if $K$ is a simplex, or $\mathcal{R}^{\ell}(K)=\mathcal{Q}^{\ell}(K)$, the space of polynomials of maximal degree $\ell$ if $K$ is a parallelogram.

Furthermore, we choose a quadrature formula $\left(\hat{x}_{j}, \hat{\omega}_{j}\right)_{j=1}^{J}$ on $\hat{K}$, where $\hat{x}_{j}$ and $\hat{\omega}_{j}$ denote the quadrature nodes and weights, respectively. To preserve the order of convergence we impose the following classical assumptions on the quadrature formula, cf. [12]

$$
\begin{array}{cl}
\hat{\omega}_{j}>0 & \forall j \in\{1,2, \ldots, J\}, \\
\sum_{j=1}^{J} \hat{\omega}_{j}\left|\nabla \hat{p}\left(\hat{x}_{j}\right)\right|^{2} \geq \hat{\lambda}\|\nabla \hat{p}\|_{L^{2}(\hat{K})} & \forall \hat{p}(\hat{x}) \in \mathcal{R}^{\ell}(\hat{K}) \text { with } \hat{\lambda}>0, \\
\int_{\hat{K}} \hat{p}(\hat{x}) d \hat{x}=\sum_{j=1}^{J} \hat{\omega}_{j} \hat{p}\left(\hat{x}_{j}\right) & \forall \hat{p}(\hat{x}) \in \mathcal{R}^{\tilde{\ell}},
\end{array}
$$

where $\tilde{\ell}=\max (2 \ell-2, \ell)$ if $\hat{K}$ is a simplicial finite element, or $\tilde{\ell}=\max (2 \ell-1, \ell+1)$ if $\hat{K}$ is a rectangular finite element. For each $K \in \mathcal{T}_{H}$ let $\left(x_{K, j}, \omega_{K, j}\right)$ denote the appropriate quadrature formula on $K$ given by $x_{K, j}=F_{K}\left(\hat{x}_{j}\right)$ and $\omega_{K, j}=\left|\operatorname{det} D F_{K}\right| \hat{\omega}_{j}$.

To achieve an arbitrary high order for the modeling error, we will use averaging kernels. These kernels were first used in the HMM context to solve highly oscillatory ordinary differential equations (see [20]). Later they were used in the FD-HMM schemes described in $[7,19]$. We recall the definition of the kernel space from [7]. A function lies in the Kernel space $\mathbb{K}^{p, q}$, if

$$
k^{(q+1)} \in B V(\mathbb{R}), \operatorname{supp} k \subset[-1,1], \text { and } \int_{\mathbb{R}} k(x) x^{r} d x= \begin{cases}1, & r=0, \\ 0, & 1 \leq r \leq p .\end{cases}
$$


The simplest averaging corresponds to a constant kernel, i.e., $k=1 / 2$ in $[-1,1]$. In this case $q=-1$.

In this work we assume that all the kernels are symmetric, i.e. $k(x)=k(-x)$ for all $x \in \mathbb{R}$. Note that this assumption is only used for the error analysis. The algorithm itself works also for nonsymmetric kernels. We will use scaled kernels given by $k_{\eta}(x)=$ $\eta^{-1} k(x / \eta)$ for $\eta>0$ with support $[-\eta, \eta]$. Furthermore, for a $d$-dimensional setting we use the same notation to mean

$$
k_{\eta}(x)=\eta^{-d} k\left(\frac{x_{1}}{\eta}\right) k\left(\frac{x_{2}}{\eta}\right) \cdots k\left(\frac{x_{d}}{\eta}\right) .
$$

As described in the introduction a FE-HMM scheme consists of a macro and a micro solver. Since the macro solver does not differ very much from the macro solver of standard FE-HMM, we will only discuss it briefly in the following subsection.

3.1. Macrosolver. The FE-HMM schemes are given for the elliptic problem (2) by

$$
\left\{\begin{array}{l}
\text { Find } u_{H} \in S_{0}^{\ell}\left(\Omega, \mathcal{T}_{H}\right) \text { such that } \\
B_{H}\left(u_{H}, v_{H}\right)=\left(F, v_{H}\right) \text { for all } v_{H} \in S_{0}^{\ell}\left(\Omega, \mathcal{T}_{H}\right),
\end{array}\right.
$$

for the heat equation (2) by

$$
\left\{\begin{aligned}
\text { Find } u_{H}:[0, T] \rightarrow S_{0}^{\ell}\left(\Omega, \mathcal{T}_{H}\right) & \text { such that } \\
\left(\partial_{t} u_{H}, v_{H}\right)+B_{H}\left(u_{H}, v_{H}\right)=\left(F, v_{H}\right) & \text { for all } v_{H} \in S_{0}^{\ell}\left(\Omega, \mathcal{T}_{H}\right), 0<t<T, \\
u_{H}(0)=f_{H} & \text { in } \Omega,
\end{aligned}\right.
$$

and for the wave equation (2) by

$$
\left\{\begin{array}{rlrl}
\text { Find } u_{H}:[0, T] \rightarrow S_{0}^{\ell}\left(\Omega, \mathcal{T}_{H}\right) & \text { such that } \\
\left(\partial_{t t} u_{H}, v_{H}\right)+B_{H}\left(u_{H}, v_{H}\right)=\left(F, v_{H}\right) & & \text { for all } v_{H} \in S_{0}^{\ell}\left(\Omega, \mathcal{T}_{H}\right), 0<t<T \\
u_{H}(0)=f_{H} & & \text { in } \Omega \\
\partial_{t} u_{H}(0)=g_{H} & & \text { in } \Omega
\end{array}\right.
$$

where the bilinear form is defined as

$$
B_{H}\left(v_{H}, w_{H}\right)=\sum_{K, j} \omega_{K, j} \int_{I_{\delta}} k_{\eta}(x) a^{\varepsilon}\left(x_{K, j}+x\right) \nabla \bar{v}_{h}(x) \cdot \nabla \bar{w}_{h}(x) d x .
$$

The summation goes over all $K \in \mathcal{T}_{H}$ and $1 \leq j \leq J$. By $\bar{v}_{h}$ and $\bar{w}_{h}$ we denote the average of the micro solution $v_{h}$ and $w_{h}$ over the sampling time interval $[-\tau, \tau]$, i.e.

$$
\bar{v}_{h}(x)=\int_{-\tau}^{\tau} k_{\tau}(t) v_{h}(t, x) d t
$$

with the obvious changes for $\bar{w}_{h}$. The micro solutions $v_{h}$ and $w_{h}$ defined on $I_{\delta}=(-\delta, \delta)^{d}$ are specified in the next section. Furthermore, $k_{\tau}$ and $k_{\eta}$ are scaled averaging kernels of a kernel $k \in \mathbb{K}^{p, q}$.

3.2. Micro solver and hyperbolic micro problem. For the micro problem we consider the FE space

$$
S^{s}\left(I_{\delta}, \mathcal{T}_{h}\right)=\left\{z_{h} \in W\left(I_{\delta}\right):\left.z_{h}\right|_{Q} \in \mathcal{R}(Q), \forall Q \in \mathcal{T}_{h}\right\},
$$


where for a periodic coupling $W\left(I_{\delta}\right)=W_{\text {per }}^{1}\left(I_{\delta}\right)$, and for a Dirichlet coupling $W\left(I_{\delta}\right)=$ $H_{0}^{1}\left(I_{\delta}\right)$. Furthermore, we use the suggestive notation $v_{h} \in v_{H, \text { lin }}+S^{s}\left(I_{\delta}, \mathcal{T}_{h}\right)$ to denote that $v_{h}-v_{H, \text { lin }} \in S^{s}\left(I_{\delta}, \mathcal{T}_{h}\right)$.

The hyperbolic micro problem reads as

$$
\left\{\begin{array}{l}
\text { Find } v_{h}:[-\tau, \tau] \rightarrow v_{H, \text { lin }}+S^{s}\left(I_{\delta}, \mathcal{T}_{h}\right) \text { such that } \\
\left(\partial_{t t} v_{h}, z_{h}\right)+\int_{I_{\delta}} a^{\varepsilon}\left(x_{K, j}+x\right) \nabla v_{h}(x) \cdot \nabla z_{h}(x) d x=0 \\
\text { for all } z_{h} \in S^{s}\left(I_{\delta}, \mathcal{T}_{h}\right),-\tau \leq t \leq \tau, \\
\quad v_{h}(0)=v_{H, \operatorname{lin}}(x) \text { and } \partial_{t} v_{h}(0)=0 \text { in } I_{\delta},
\end{array}\right.
$$

where $v_{H, \operatorname{lin}}(x)=v_{H}\left(x_{K, j}\right)+\nabla v_{H}\left(x_{K, j}\right) \cdot x$. From the definition it is clear that $v_{h}$ and thus $\bar{v}_{h}$ depend on $K$ and $j$. For the ease of reading we do not reflect this dependence in the notation. Note that we center the sampling domain for every quadrature node $x_{K, j}$ at the origin. This shift is taken into account be evaluating $a^{\varepsilon}$ at $x_{K, j}+x$. Using sampling domains centered at $x_{K, j}$ is also possible and would lead to an equivalent scheme. Our choice, however, simplifies the subsequent analysis a little, since fewer shifts are needed.

We would like to emphasize again that the use of a hyperbolic micro problem is the crucial difference between the FE-HMM we propose and the former FE-HMM that uses an elliptic micro problem instead. Since the wave equation and its FEM approximation provide a finite speed of propagation, the influence of the boundary condition vanishes by choosing $\delta$ such that no information may travel from boundary into the averaging domain $[-\eta, \eta]^{d}$. Hence, the choice of the boundary condition has only a minor relevance compared with standard FE-HMM, see [26]. The convergence results in the next section hold for periodic and for Dirichlet coupling condition as well.

\section{Error analysis}

The analysis of FE-HMM schemes relies in a decomposition of the overall error into a macro, a micro, and a modeling error. For this decomposition we introduce two additional bilinear forms. Firstly, the discretized homogenized bilinear form is given by

$$
B_{H}^{0}\left(v_{H}, w_{H}\right)=\sum_{K \in \mathcal{T}_{H}} \sum_{j=1}^{J} \omega_{K, j} a^{0}\left(x_{K, j}\right) \nabla v_{H}\left(x_{K, j}\right) \cdot \nabla w_{H}\left(x_{K, j}\right) .
$$

This corresponds to a classical FEM approximation of the homogenized equation with numerical quadrature. However, since the homogenized tensor $a^{0}$ is usually not known a priori we can not use it in an actual computation. Note that in addition we compute neither $a^{0}$ nor $B^{0}$ with our FE-HMM scheme. They are used only for the error analysis. Secondly, the semidiscrete FE-HMM formulation is given by

$$
\tilde{B}_{H}\left(v_{H}, w_{H}\right)=\sum_{K, j} \omega_{K, j} \int_{I_{\delta}} k_{\eta}(x) a^{\varepsilon}\left(x_{K, j}+x\right) \nabla \bar{v}(x) \cdot \nabla \bar{w}(x) d x,
$$

where $\bar{v}$ and $\bar{w}$ are the time averages of the solutions of the non-discretized counterpart of the micro problem. In more detail, we have

$$
\bar{v}(x)=\int_{-\tau}^{\tau} k_{\tau}(t) v(t, x) d t
$$


where $v$ solves

$$
\left\{\begin{array}{c}
\text { Find } v:[-\tau, \tau] \rightarrow v_{H, \text { lin }}+W\left(I_{\delta}\right) \text { such that } \\
\left(\partial_{t t} v, z\right)+\int_{I_{\delta}} a^{\varepsilon}\left(x_{K, j}+x\right) \nabla v \cdot \nabla z d x=0 \\
\text { for all } z \in W\left(I_{\delta}\right),-\tau \leq t \leq \tau \\
v(0)=v_{H, \text { lin }}(x) \text { and } \partial_{t} v(0)=0 \text { in } I_{\delta} .
\end{array}\right.
$$

Clearly, $\bar{w}$ is defined accordingly. Again, due to the finite speed of propagation the boundary condition does not influence $\tilde{B}_{H}$ if $\delta$ is chosen sufficiently large compared to $\eta$ and $\tau$. By sufficiently large, we mean that

$$
\delta \geq \eta+\tau \sqrt{\beta}
$$

where $\beta$ is the boundedness constant of $a^{\varepsilon}$, see (2). Due to the finite speed of propagation of waves, the solution inside the region $I_{\eta} \times(-\tau, \tau)$ where the local averaging takes place, will not be affected by the boundary conditions imposed on the boundary of $I_{\delta}$ if (4) holds. In this case, the micro problem (4) can be replaced by the following infinite domain problem without any influence on $\tilde{B}_{H}$.

$$
\left\{\begin{aligned}
\partial_{t t} v(t, x) & =\nabla \cdot\left(a^{\varepsilon}(x) \nabla v(t, x)\right) & & \text { in } \mathbb{R}^{d},-\tau \leq t \leq \tau \\
v(0, x) & =v_{H, \operatorname{lin}}(x) & & \text { in } \mathbb{R}^{d} \\
\partial_{t} v(0, x) & =0 & & \text { in } \mathbb{R}^{d} .
\end{aligned}\right.
$$

This formulation simplifies the error analysis below, since we do no longer have to deal with boundary conditions for the micro problem.

By $u_{H}^{0}$ we denote the FEM approximation of the analytically homogenized problem and by $\tilde{u}_{H}$ the solution of the semidiscrete FE-HMM. To be more precise, $u_{H}^{0}$ is the solution to $(3.1),(3.1)$, or $(3.1)$, where $B_{H}$ is replaced with $B_{H}^{0}$, and $\tilde{u}_{H}$ is the solution of the corresponding equations, where $B_{H}$ is replaced with $\tilde{B}_{H}$. The convergence proof of the FE-HMM can now be outlined as follows.

Due to the triangle inequality we have

$$
\left\|u^{0}-u_{H}\right\| \leq \underbrace{\left\|u^{0}-u_{H}^{0}\right\|}_{=e_{\mathrm{MAC}}}+\underbrace{\left\|u_{H}^{0}-\tilde{u}_{H}\right\|}_{=e_{\mathrm{MOD}}}+\underbrace{\left\|\tilde{u}_{H}-u_{H}\right\|}_{=e_{\mathrm{MIC}}},
$$

The macro error $e_{\mathrm{MAC}}$ can be bounded using standard convergence results for FEM with numerical integration. However, to estimate the modeling error $e_{\mathrm{MOD}}$ and the micro error $e_{\mathrm{MIC}}$ we use Strang-type lemmas.

4.1. Strang-type lemmas. Strang-type lemmas were originally considered to handle nonconforming FEM arising by the use of quadrature formulas to evaluate integrals in standard FEM; see [9, 11,24]. While the elliptic version can be considered as a classical result, generalized versions for the heat and the wave equation were only proven recently. Here we summarize these results in a unified setting.

For the following three Strang-type lemmas let $V$ be a closed subset of $H_{0}^{1}(\Omega)$ and $f, g \in V$ be given as above. We assume that the two bilinear forms $B_{1}$ and $B_{2}$ on $V$ are coercive and bounded, i.e., there are $0<\tilde{\alpha} \leq \tilde{\beta}$ such that

$$
\tilde{\alpha}\|v\|_{H^{1}(\Omega)}^{2} \leq B_{i}(v, v) \text { and }\left|B_{i}(v, w)\right| \leq \tilde{\beta}\|v\|_{H^{1}(\Omega)}\|w\|_{H^{1}(\Omega)},
$$


for $i=1,2$ and for all $v, w \in V$. Furthermore, we introduce the difference over $V$ between two bilinear forms given by

$$
d_{V}\left(B_{1}, B_{2}\right)=\sup _{v, w \in V \backslash\{0\}} \frac{\left|B_{1}(v, w)-B_{2}(v, w)\right|}{\|v\|_{H^{1}(\Omega)}\|w\|_{H^{1}(\Omega)}} .
$$

The classical elliptic Strang-type lemma can now be written as follows.

Lemma 4.1. Let $F \in L^{2}(\Omega)$ and $u_{1}, u_{2}$ be the unique solutions of

$$
\left\{\begin{array}{l}
\text { Find } u_{1} \in V, \text { such that } \\
B_{1}\left(u_{1}, v\right)=(F, v) \quad \forall v \in V,
\end{array}\right.
$$

and

$$
\left\{\begin{array}{l}
\text { Find } u_{2} \in V, \text { such that } \\
B_{2}\left(u_{2}, v\right)=(F, v) \quad \forall v \in V .
\end{array}\right.
$$

Assume that $u_{i} \in H^{1}(\Omega)$ for $i=1,2$. Then

$$
\left\|u_{1}-u_{2}\right\|_{H^{1}(\Omega)} \leq C d_{V}\left(B_{1}, B_{2}\right)
$$

where $C$ depends on $\left\|u_{i}\right\|_{H^{1}(\Omega)}$, but is independent of $d_{V}\left(B_{1}, B_{2}\right)$.

The version of the Strang-type lemma for the heat equation in [6] is more general, since the bilinear form may also depend on time. Here we simplify the result by only considering time-independent coefficients $a^{\varepsilon}$, i.e., bilinear forms that are constant in time.

Lemma 4.2. For given $T>0$ let $F \in L^{2}\left(0, T ; L^{2}(\Omega)\right)$ and $u_{1}, u_{2}$ be the unique solutions of

$$
\left\{\begin{aligned}
\text { Find } u_{1}:[0, T] \rightarrow V, \text { such that } & & \\
\left(\partial_{t} u_{1}(t), v\right)+B_{1}\left(u_{1}(t), v\right)=(F(t), v) & & \forall v \in V \Omega, 0<t<T, \\
u_{1}(0)=f & & \text { in } \Omega,
\end{aligned}\right.
$$

and

$$
\left\{\begin{aligned}
\text { Find } u_{2}:[0, T] \rightarrow V, \text { such that } & \\
\left(\partial_{t} u_{2}(t), v\right)+B_{2}\left(u_{2}(t), v\right)=(F(t), v) & \forall v \in V \Omega, 0<t<T, \\
u_{2}(0)=f & \text { in } \Omega .
\end{aligned}\right.
$$

Assume that $\partial_{t}^{k} u_{i} \in L^{2}\left(0, T ; H^{1}(\Omega)\right)$ for $k=0,1$ and $i=1,2$. Then

$$
\left\|u_{1}-u_{2}\right\|_{L^{\infty}\left(0, T ; H^{1}(\Omega)\right)} \leq C d_{V}\left(B_{1}, B_{2}\right) .
$$

where $C$ depends on $\left\|\partial_{t}^{k} u_{i}\right\|_{L^{2}\left(0, T ; H^{1}(\Omega)\right)}, k=0,1$, but is independent of $d_{V}\left(B_{1}, B_{2}\right)$.

For the wave equation we finally have the following version from [4].

Lemma 4.3. For given $T>0$ let $F, \partial_{t} F \in L^{2}\left(0, T ; L^{2}(\Omega)\right)$ and $u_{1}, u_{2}$ be the unique solutions of

$$
\left\{\begin{array}{rlrl}
\text { Find } u_{1}:[0, T] \rightarrow V, & \text { such that } & & \\
\left(\partial_{t t} u_{1}(t), v\right)+B_{1}\left(u_{1}(t), v\right) & =(F(t), v) & & \forall v \in V \Omega, 0<t<T, \\
u_{1}(0) & =f & & \text { in } \Omega, \\
\partial_{t} u_{1}(0)=g & & \text { in } \Omega,
\end{array}\right.
$$


and

$$
\left\{\begin{aligned}
\text { Find } u_{2}:[0, T] \rightarrow V, \text { such that } & & \\
\left(\partial_{t t} u_{2}(t), v\right)+B_{2}\left(u_{2}(t), v\right)=(F(t), v) & & \forall v \in V \Omega, 0<t<T, \\
u_{2}(0)=f & & \text { in } \Omega, \\
\partial_{t} u_{2}(0)=g & & \text { in } \Omega .
\end{aligned}\right.
$$

Assume that $\partial_{t}^{k} u_{i} \in L^{2}\left(0, T ; H^{1}(\Omega)\right)$ for $k=0,1,2$ and $i=1,2$. Then

$$
\left\|\partial_{t}\left(u_{1}-u_{2}\right)\right\|_{L^{\infty}\left(0, T ; L^{2}(\Omega)\right)}+\left\|u_{1}-u_{2}\right\|_{L^{\infty}\left(0, T ; H^{1}(\Omega)\right)} \leq C d_{V}\left(B_{1}, B_{2}\right),
$$

where $C$ depends on $\left\|\partial_{t}^{k} u_{i}\right\|_{L^{2}\left(0, T ; H^{1}(\Omega)\right)}, k=0,1,2$, but is independent of $d_{V}\left(B_{1}, B_{2}\right)$.

Combining these lemmas with (4) we get

$$
\left\|u^{0}-u_{H}\right\| \leq \underbrace{\left\|u^{0}-u_{H}^{0}\right\|}_{=e_{\mathrm{MAC}}}+\underbrace{C d_{V}\left(B_{H}^{0}, \tilde{B}_{H}\right)}_{=e_{\mathrm{MOD}}}+\underbrace{C d_{V}\left(\tilde{B}_{H}, B_{H}\right)}_{=e_{\mathrm{MIC}}},
$$

where $\|\cdot\|$ denotes the norm specified in the lemmas 4.1 , respectively 4.2 or 4.3 . Moreover, the space $V$ equals here the macro FE space $S^{\ell}\left(\Omega, \mathcal{T}_{H}\right)$. The remaining principal task is now to bound the differences between the bilinear forms. However, since $B_{H}$ relies on averaging kernels, we will first recall some of their useful properties.

4.2. Averaging kernels. The computation of the bilinear form in FE-HMM involves averaging a highly oscillatory function over the microscopic domain $I_{\delta}$. For a fixed oscillation length $\varepsilon$, the high frequencies of the oscillatory function is filtered out by using an averaging operator. The use of general averaging kernels allows us to achieve high order convergence rates in terms of $\varepsilon / \eta$, where $\eta<\delta$ represents the size of the support of the kernel. Now we present a lemma, namely [8, Lemma 2.3], which shows that this averaging can be made as accurate as we like. Here we cite it in a simplified version adapted to our setting.

LEMMA 4.4. Let $\varphi$ be a 1-periodic continuous function and denote its average over one period by $\bar{\varphi}=\int_{0}^{1} \varphi(t) d t$. Then we have for $k \in \mathbb{K}^{p, q}$

$$
\left|\int_{\mathbb{R}} k_{\eta}(t) \varphi\left(\frac{t}{\varepsilon}\right) d t-\bar{\varphi}\right| \leq C\|\varphi\|_{L^{\infty}(\mathbb{R})}\left(\frac{\varepsilon}{\eta}\right)^{q+2},
$$

where the constant $C$ does not depend on $\varepsilon, \eta, \varphi$.

Later in the analysis we need to apply the averaging operator to functions in many dimensions. Later in the analysis we will use the corollary, which generalizes Lemma 4.4 to higher dimensions. The result follows by a simple induction over the dimension $d$.

Corollary 4.5. Let $\Phi$ be a $Y$-periodic continuous function and denote its average over one period by $\bar{\Phi}=\int_{Y} \Phi(x) d x$. Then, we have for $k \in \mathbb{K}^{p, q}$

$$
\int_{\mathbb{R}^{d}} k_{\eta}(x) \Phi\left(\frac{x}{\varepsilon}\right) d x=\bar{\Phi}+\gamma\left(\frac{\varepsilon}{\eta}\right)^{q+2},
$$

where $|\gamma| \leq C\|\Phi\|_{L^{\infty}\left(\mathbb{R}^{d}\right)}$ and $C$ is independent of $\varepsilon$, $\eta$, and $\Phi$. 
To compute the FE-HMM bilinear form $B_{H}$, we need the local time average of the solution of a second order wave equation in the sampling domain $I_{\delta}$. In $[8$, Lemma 2.3, Theorem 4.1], the authors proved that the time averages of solutions of second order wave equations with periodic coefficients solve PDEs of elliptic type. The righthand side of the resulting elliptic PDE converges to the time average of the right-hand side of the hyperbolic PDE with $O(\varepsilon / \tau)^{q}$ convergence rate. Here, we provide a lemma which improves the convergence rate to $O(\varepsilon / \tau)^{q+2}$ when the right-hand side of the wave equation is time-independent.

LEMma 4.6. Assume that $a \in\left(C^{\infty}\left(\mathbb{R}^{d}\right)\right)^{d \times d}$ is $Y$-periodic, symmetric, and uniformly elliptic and bounded (cf. (2)). Furthermore let $\Phi \in C^{\infty}\left(\mathbb{R}^{d}\right)$ be a $Y$-periodic function with $\bar{\Phi}=0, k \in \mathbb{K}^{p, q}$ with an even $q$, and $w$ the solution of the problem

$$
\left\{\begin{aligned}
\partial_{t t} w(t, x) & =\nabla \cdot(a(x) \nabla w(t, x))+\Phi(x), & & \text { for } x \in \mathbb{R}^{d}, t>0 \\
w(0, x) & =0, & & \text { for } x \in \mathbb{R}^{d} \\
\partial_{t} w(0, x) & =0, & & \text { for } x \in \mathbb{R}^{d} .
\end{aligned}\right.
$$

Let the local time average $\bar{w}$ be defined as

$$
\bar{w}(x):=\int_{\mathbb{R}} k_{\tau}(t) w\left(\frac{t}{\varepsilon}, x\right) d t .
$$

Then for $0<\varepsilon \leq \tau$ the local time average $\bar{w}$ satisfies

$$
\nabla \cdot(a(x) \nabla \bar{w}(x))=-\Phi(x)+\left(\frac{\varepsilon}{\tau}\right)^{q+2} R(x),
$$

where $R$ is $Y$-periodic with zero average, i.e. $\bar{R}=0$, and

$$
\|R\|_{H^{1}(Y)} \leq C\|\Phi\|_{L^{2}(Y)} .
$$

Proof. We represent by $\left\{\lambda_{j}^{2}, \varphi_{j}\right\}$ the eigenvalue and eigenvector pair of the operator $L:=-\nabla \cdot(a \nabla)$. The eigenvalues are strictly positive except for $\lambda_{0}^{2}=0$, and the eigenvectors $\left\{\varphi_{j}\right\}_{j=0}^{\infty}$ are smooth periodic functions such that

$$
\bar{\varphi}_{j}= \begin{cases}1, & j=0 \\ 0, & j>0\end{cases}
$$

Moreover, the eigenfunctions form an orthonormal basis for the periodic functions in $L^{2}(Y)$ (see [22]). Now, we start with an eigenfunction expansion of the terms $w(t, x)$ and $\Phi(x)$. Since $a$ and $\Phi$ are periodic, $w$ will also be periodic. Furthermore, by the zero initial data of $w$ we conclude that $\overline{w(t, \cdot)}=0$. Hence,

$$
w(t, x)=\sum_{j=1}^{\infty} w_{j}(t) \varphi_{j}(x), \quad \Phi(x)=\sum_{j=1}^{\infty} \phi_{j} \varphi_{j}(x) .
$$

We use the orthogonality of the eigenvectors and put the above expressions into the wave equation. This gives

$$
\left\{\begin{array}{c}
w_{j}^{\prime \prime}(t)+\lambda_{j}^{2} w_{j}(t)=\phi_{j} \\
w_{j}(0)=w_{j}^{\prime}(0)=0 .
\end{array}\right.
$$


Since $\phi_{j}$ is a constant, we can write the solution $w_{j}$ explicitly as

$$
w_{j}(t)=\frac{\phi_{j}}{\lambda_{j}^{2}}\left(1-\cos \left(\lambda_{j} t\right)\right)
$$

Now we define

$$
c_{j}:=\left(\frac{\varepsilon}{\tau}\right)^{-q-2} \int_{\mathbb{R}} \kappa_{\tau}(t) \cos \left(\frac{\lambda_{j} t}{\varepsilon}\right) d t .
$$

Then

$$
\bar{w}(x)=\sum_{j=1}^{\infty} \frac{\phi_{j}}{\lambda_{j}^{2}} \varphi_{j}(x)-\left(\frac{\varepsilon}{\tau}\right)^{q+2} \sum_{j=1}^{\infty} \frac{\phi_{j}}{\lambda_{j}^{2}} c_{j} \varphi_{j}(x)
$$

and

$$
\begin{aligned}
L[\bar{w}](x) & =\sum_{j=1}^{\infty} \frac{\phi_{j}}{\lambda_{j}^{2}} L\left[\varphi_{j}\right](x)-\left(\frac{\varepsilon}{\tau}\right)^{q+2} L\left[\sum_{j=1}^{\infty} \frac{\phi_{j}}{\lambda_{j}^{2}} c_{j} \varphi_{j}\right](x) \\
& =\sum_{j=1}^{\infty} \phi_{j} \varphi_{j}(x)+\left(\frac{\varepsilon}{\tau}\right)^{q+2} R(x)=\Phi(x)+\left(\frac{\varepsilon}{\tau}\right)^{q+2} R(x),
\end{aligned}
$$

where

$$
R(x)=L\left[\sum_{j=1}^{\infty} \frac{\phi_{j}}{\lambda_{j}^{2}} c_{j} \varphi_{j}\right](x)
$$

To get an $H^{1}$ estimate of $R$, we use the fact that $q$ is even and apply the operator $L^{q / 2+1}$ to $R$. This gives

$$
L^{q / 2+1}[R]=\sum_{j=1}^{\infty} \phi_{j} c_{j} \lambda_{j}^{q+2} \varphi_{j}(x)
$$

Then by standard elliptic regularity and Parseval's identity it follows that

$$
\|R\|_{H^{1}(Y)}^{2} \leq\|R\|_{H^{q+2}(Y)}^{2} \leq C\left\|\sum_{j=1}^{\infty} \phi_{j} c_{j} \lambda_{j}^{q+2} \varphi_{j}\right\|_{L^{2}(Y)}^{2}=C\left|\sum_{j=1}^{\infty} \phi_{j}^{2} c_{j}^{2} \lambda_{j}^{2 q+4}\right| .
$$

Moreover, by Lemma 4.4 we get

$$
\left|c_{j}\right| \leq\left(\frac{\varepsilon}{\tau}\right)^{-q-2} C^{\prime}\left(\frac{\varepsilon}{\tau \lambda_{j}}\right)^{q+2}=C^{\prime} \frac{1}{\lambda_{j}^{q+2}},
$$

where $C^{\prime}$ does not depend on $\lambda_{j}, \tau, \varepsilon$. From here, we obtain

$$
\|R\|_{H^{1}(Y)}^{2} \leq C C^{\prime}\left(\sum_{j=1}^{\infty} \phi_{j}^{2}\right)^{2} \leq \tilde{C}\|\Phi\|_{L^{2}(Y)}^{2} .
$$

Note that $\tilde{C}$ may depend on $p$ and $q$, but not on $\tau$ or $\varepsilon$. 
4.3. Modeling error. In this section, we state our main result. In particular, we prove that the modeling error defined in (4) can be made arbitrarily small by using a kernel with higher $q$, i.e., higher regularity properties.

TheOREM 4.7. Let $B_{H}^{0}$ and $\tilde{B}_{H}$ be given by (4) and (4), respectively. Suppose that the averaging kernel $k$ used in the definition of $\tilde{B}_{H}$ lies in $\mathbb{K}^{p, q}$ with even $q, \eta=\tau$, and $\delta \geq \eta+\tau \sqrt{\beta}$. Furthermore, let $a^{\varepsilon}(x)$ be given by $a^{\varepsilon}(x)=a(x / \varepsilon)$, where $a \in\left(C^{\infty}\left(\mathbb{R}^{d}\right)\right) d \times d$ is $Y$-periodic, symmetric, elliptic, and bounded (cf. (2)). Then

$$
d_{V}\left(B_{H}^{0}, \tilde{B}_{H}\right) \leq C\left(\frac{\varepsilon}{\eta}\right)^{q+2}
$$

where $V=S^{\ell}\left(\Omega, \mathcal{T}_{H}\right)$ and $C$ is a constant independent of $\varepsilon$ and $\eta$.

Proof. Our first goal is to rewrite $\bar{v}$ (resp. $\bar{w}$ ) defined in (4) in terms of $v_{H}$ and the periodic continuations of the solutions $\chi^{i}$ of the cell problem $(2.1)$, which we denoted by $\chi^{i}$ as well. Afterwards, we insert this reformulation into the definition of the bilinear form, $\tilde{B}_{H}$, of the semidiscrete FE-HMM formulation.

Due to the assumption on $\delta$, the micro problem (4) can be replaced by the infinite domain problem (4) without any influence on $\tilde{B}_{H}$, as explained earlier. We denote the solution of (4) by $v$ and introduce its scaled and adjusted counterpart

$$
V(t, x)=\varepsilon^{-1}(v(t \varepsilon, x \varepsilon)-v(0, x \varepsilon)) .
$$

We see that $V$ satisfies

$$
\left\{\begin{aligned}
\partial_{t t} V(t, x) & =\nabla \cdot\left(a(x)\left(\nabla V(t, x)+\nabla v_{H}\left(x_{K, j}\right)\right)\right. & & \text { in } \mathbb{R}^{d},-\tau \leq t \leq \tau, \\
V(0, x) & =0 & & \text { in } \mathbb{R}^{d}, \\
\partial_{t} V(0, x) & =0 & & \text { in } \mathbb{R}^{d},
\end{aligned}\right.
$$

and consider the time average of $V$

$$
\bar{V}(x)=\int_{-\tau}^{\tau} k_{\tau}(t) V\left(\frac{t}{\varepsilon}, x\right) d t .
$$

Now, we can apply Lemma 4.6 with the identifications

$$
w=V \text { and } \Phi=\nabla \cdot\left(a(x) \nabla v_{H}\left(x_{K, j}\right)\right) .
$$

Formula (4.6) in Lemma 4.6 immediately gives

$$
\nabla \cdot(a(x) \nabla \bar{V}(x))=-\nabla \cdot\left(a(x) \nabla v_{H}\left(x_{K, j}\right)\right)+\left(\frac{\varepsilon}{\tau}\right)^{q+2} R_{v}(x) .
$$

Furthermore, we can rewrite the time average as

$$
\bar{V}(x)=\sum_{i=1}^{d} \chi^{i}(x) \partial_{x_{i}} v_{H}\left(x_{K, j}\right)+\left(\frac{\varepsilon}{\tau}\right)^{q+2} e_{v}(x),
$$

where $e_{v}$ is the zero average solution of the periodic problem

$$
\nabla \cdot\left(a(x) \nabla e_{v}(x)\right)=R_{v}(x)
$$


Inserting $v$ from (4.3) into (4) we get

$$
\begin{aligned}
\bar{v}(x) & =\varepsilon \int_{-\tau}^{\tau} k_{\tau} V\left(\frac{t}{\varepsilon}, \frac{x}{\varepsilon}\right) d t+v(0, x) \\
& =v_{H, \operatorname{lin}}(x)+\varepsilon \sum_{i=1}^{d} \chi^{i}\left(\frac{x}{\varepsilon}\right) \partial_{x_{i}} v_{H}\left(x_{K, j}\right)+\varepsilon\left(\frac{\varepsilon}{\tau}\right)^{q+2} e_{v}\left(\frac{x}{\varepsilon}\right) .
\end{aligned}
$$

To simplify the subsequent analysis we use the abbreviation

$$
v_{H}^{*}(x)=v_{H, \operatorname{lin}}(x)+\varepsilon \sum_{i=1}^{d} \chi^{i}\left(\frac{x}{\varepsilon}\right) \partial_{x_{i}} v_{H}\left(x_{K, j}\right) .
$$

Therefore,

$$
\nabla \bar{v}(x)=\nabla v_{H}^{*}(x)+\left(\frac{\varepsilon}{\tau}\right)^{q+2} \nabla e_{v}\left(\frac{x}{\varepsilon}\right)
$$

and

$$
\nabla v_{H}^{*}(x)=\nabla v_{H}\left(x_{K, j}\right)+\sum_{i=1}^{d} \nabla \chi^{i}\left(\frac{x}{\varepsilon}\right) \partial_{x_{i}} v_{H}\left(x_{K, j}\right) .
$$

Here $\nabla e_{v}(x / \varepsilon)=\left.\nabla_{y} e_{v}(y)\right|_{y=x / \varepsilon}$ and $\nabla \chi^{i}(x / \varepsilon)$ should be interpreted similarly. Note that due to the periodicity of $\chi^{i}$, the gradient of $v_{H}^{*}$ is $\varepsilon Y$-periodic as well. With obvious changes, the above considerations hold for $w_{H}$ as well. We insert (4.3) into (4) to get

$$
\tilde{B}_{H}\left(v_{H}, w_{H}\right)=\tilde{B}_{H}^{1}\left(v_{H}, w_{H}\right)+\left(\frac{\varepsilon}{\tau}\right)^{q+2} \tilde{B}_{H}^{2}\left(v_{H}, w_{H}\right)
$$

where

$$
\tilde{B}_{H}^{1}\left(v_{H}, w_{H}\right)=\sum_{K, j} \omega_{K, j} \int_{I_{\delta}} k_{\eta}(x) a^{\varepsilon}\left(x_{K, j}+x\right) \nabla v_{H}^{*}(x) \cdot \nabla w_{H}^{*}(x) d x
$$

and

$$
\begin{aligned}
\tilde{B}_{H}^{2}\left(v_{H}, w_{H}\right)= & \sum_{K, j} \omega_{K, j} \int_{I_{\delta}} k_{\eta}(x) a^{\varepsilon}\left(x_{K, j}+x\right) \nabla v_{H}^{*}(x) \cdot \nabla e_{w}\left(\frac{x}{\varepsilon}\right) d x \\
& +\sum_{K, j} \omega_{K, j} \int_{I_{\delta}} k_{\eta}(x) a^{\varepsilon}\left(x_{K, j}+x\right) \nabla w_{H}^{*}(x) \cdot \nabla e_{v}\left(\frac{x}{\varepsilon}\right) d x \\
& +\left(\frac{\varepsilon}{\tau}\right)^{q+2} \sum_{K, j} \omega_{K, j} \int_{I_{\delta}} k_{\eta}(x) a^{\varepsilon}\left(x_{K, j}+x\right) \nabla e_{v}\left(\frac{x}{\varepsilon}\right) \cdot \nabla e_{w}\left(\frac{x}{\varepsilon}\right) d x
\end{aligned}
$$

We will now bound $\tilde{B}_{H}^{1}$. Since $a^{\varepsilon}, \nabla w_{H}^{*}$, and $\nabla v_{H}^{*}$ are $\varepsilon Y$-periodic, we can apply Corollary 4.5 to get

$$
\begin{aligned}
\tilde{B}_{H}^{1}\left(v_{H}, w_{H}\right) & =\sum_{K, j} \omega_{K, j} \int_{Y} a(x) \nabla v_{H}^{*}(\varepsilon x) \cdot \nabla w_{H}^{*}(\varepsilon x) d x+\left(\frac{\varepsilon}{\eta}\right)^{q+2} \sum_{K, j} \omega_{K, j} \gamma_{K, j} \\
& =\sum_{K, j} \omega_{K, j} a^{0} \nabla v_{H}\left(x_{K, j}\right) \cdot \nabla w_{H}\left(x_{K, j}\right)+\left(\frac{\varepsilon}{\eta}\right)^{q+2} \sum_{K, j} \omega_{K, j} \gamma_{K, j} \\
& =B_{H}^{0}\left(v_{H}, w_{H}\right)+\left(\frac{\varepsilon}{\eta}\right)^{q+2} \sum_{K, j} \omega_{K, j} \gamma_{K, j} .
\end{aligned}
$$


In the second equality we used (4.3) and the formula (2.1) for the homogenized matrix. From Corollary 4.5 we have that the constants $\gamma_{K, j}$ are bounded by

$$
\begin{aligned}
\left|\gamma_{K, j}\right| & \leq C\left\|a(x) \nabla v_{H}^{*}(\varepsilon x) \cdot \nabla w_{H}^{*}(\varepsilon x)\right\|_{L^{\infty}\left(\mathbb{R}^{d}\right)} \\
& \leq C\left|\nabla v_{H}\left(x_{K, j}\right)\right|\left|\nabla w_{H}\left(x_{K, j}\right)\right|,
\end{aligned}
$$

where we absorbed the contribution of $a$ and $\chi^{i}$ into the constant $C$. Then by the Cauchy-Schwartz inequality, by the assumption (3) on the quadrature formula, and the Poincaré inequality it follows that

$$
\begin{aligned}
\left|\sum_{K, j} \omega_{K, j} \gamma_{K, j}\right| & \leq C \sum_{K, j} \omega_{K, j}\left|\nabla v_{H}\left(x_{K, j}\right)\right|\left|\nabla w_{H}\left(x_{K, j}\right)\right| \\
& \leq C\left(\sum_{K, j} \omega_{K, j}\left|\nabla v_{H}\left(x_{K, j}\right)\right|^{2}\right)^{1 / 2}\left(\sum_{K, j} \omega_{K, j}\left|\nabla w_{H}\left(x_{K, j}\right)\right|^{2}\right)^{1 / 2} \\
& \leq C\left\|v_{H}\right\|_{H^{1}(\Omega)}\left\|w_{H}\right\|_{H^{1}(\Omega)} .
\end{aligned}
$$

Hence we have

$$
\tilde{B}_{H}^{1}\left(v_{H}, w_{H}\right)=B_{H}^{0}\left(v_{H}, w_{H}\right)+C\left(\frac{\varepsilon}{\eta}\right)^{q+2}\left\|v_{H}\right\|_{H^{1}(\Omega)}\left\|w_{H}\right\|_{H^{1}(\Omega)} .
$$

It remains to show, that $\tilde{B}_{H}^{2}$ is bounded from above. Here we show the estimate of the last term in $\tilde{B}_{H}^{2}$. The proof of the first two terms in $\tilde{B}_{H}^{2}$ follows similarly. First we use Corollary 4.5 to see that

$$
\begin{aligned}
& \sum_{K, j} \omega_{K, j} \int_{I_{\delta}} K_{\eta}(x) a^{\varepsilon}(x) \nabla e_{v}\left(\frac{x}{\varepsilon}\right) \cdot \nabla e_{w}\left(\frac{x}{\varepsilon}\right) d x \\
= & \sum_{K, j} \omega_{K, j} \int_{Y} a(y) \nabla e_{v}(y) \cdot \nabla e_{w}(y) d y+\left(\frac{\varepsilon}{\eta}\right)^{q+2} \sum_{K \in \mathcal{T}_{H}} \sum_{j=1}^{J} \omega_{K, j} \gamma_{K, j} .
\end{aligned}
$$

Then by elliptic regularity of equation (4.3) and by the estimate (4.6) in Lemma 4.6 we get

$$
\left\|e_{v}\right\|_{H^{r+2}(Y)} \leq C\left\|R_{v}\right\|_{H^{r}(Y)} \leq C\left|\nabla v_{H}\left(x_{K, j}\right)\right|, \quad r=0,1
$$

Therefore

$$
\begin{aligned}
\left|\int_{Y} a(y) \nabla e_{v}(y) \cdot \nabla e_{w}(y) d y\right| & \leq\left\|a \nabla e_{v}\right\|_{L^{2}(Y)}\left\|\nabla e_{w}\right\|_{L^{2}(Y)} \\
& \leq C\left\|e_{w}\right\|_{H^{1}(Y)}\left\|e_{v}\right\|_{H^{1}(Y)} \\
& \leq C\left|\nabla v_{H}\left(x_{K, j}\right)\right|\left|\nabla w_{H}\left(x_{K, j}\right)\right| .
\end{aligned}
$$

Moreover, if $d=1,2,3$ then $H^{r}(Y) \hookrightarrow C(Y)$ for $r>d / 2 \geq 1.5$. Hence, with $r=2$ we have

$$
\begin{aligned}
\left|\gamma_{K, j}\right| & \leq\left|a \nabla e_{v} \cdot \nabla e_{w}\right|_{\infty} \leq\left|a \nabla e_{v}\right|_{\infty} \cdot\left|\nabla e_{w}\right|_{\infty} \leq C\left\|a \nabla e_{v}\right\|_{H^{2}(Y)}\left\|\nabla e_{w}\right\|_{H^{2}(Y)} \\
& \leq C\left\|e_{v}\right\|_{H^{3}(Y)}\left\|e_{w}\right\|_{H^{3}(Y)} \leq C\left|\nabla v_{H}\left(x_{K, j}\right)\right|\left|\nabla w_{H}\left(x_{K, j}\right)\right|
\end{aligned}
$$


4.4. Fully discrete a priori error analysis. Combining the previous results, we eventually get the following convergence result for the FE-HMM with hyperbolic micro problems.

THEOREM 4.8. Suppose that $u^{0}$ is the solution of the homogenized problems (2.1), (2.1), or (2.1) and $u_{H}$ the corresponding FE-HMM solutions solving (3.1), (3.1), or (3.1). Suppose that the assumptions of Theorem 4.7 hold. Then

$$
\left\|u^{0}-u_{H}\right\| \leq C\left(H^{\ell}+\left(\frac{\varepsilon}{\eta}\right)^{q+2}+\left(\frac{h}{\varepsilon}\right)^{s}\right),
$$

where $C$ does not depend on $\varepsilon, \eta, h$, or $H$. Note that the norm in the statement is the same norm as in the corresponding Strang-type lemmas 4.1, 4.2, or 4.3.

Proof. As mentioned before, we start by splitting the error into three parts and apply the Strang type lemmas $4.1,4.2$, and 4.3 to the latter two. Thus, we have

$$
\begin{aligned}
\left\|u^{0}-u_{H}\right\| & \leq\left\|u^{0}-u_{H}^{0}\right\|+\left\|u_{H}^{0}-\tilde{u}_{H}\right\|+\left\|\tilde{u}_{H}-u_{H}\right\| \\
& \leq\left\|u^{0}-u_{H}^{0}\right\|+C d_{V}\left(B_{H}^{0}, \tilde{B}_{H}\right)+C d_{V}\left(\tilde{B}_{H}, B_{H}\right) .
\end{aligned}
$$

While the first term, i.e. the macro error, can be bounded by classical results for FEM $[9,11,24]$, we use Theorem 4.7 to bound the second one. Hence, it remains to bound the last term which accounts for the micro error. To simplify the notation we denote by

$$
B_{K, j}(v, w)=\int_{I_{\delta}} k_{\eta}(x) a^{\varepsilon}\left(x_{K, j}+x\right) \nabla v \cdot \nabla w d x
$$

the contribution corresponding to quadrature node $x_{K, j}$ to the FE-HMM bilinear form. The discrete and the semidiscrete FE-HMM bilinear form can thereby be rewritten as

$$
B_{H}\left(v_{H}, w_{H}\right)=\sum_{K, j} \omega_{K, j} B_{K, j}\left(\bar{v}_{h}, \bar{w}_{h}\right) \quad \text { and } \quad \tilde{B}_{H}\left(v_{H}, w_{H}\right)=\sum_{K, j} \omega_{K, j} B_{K, j}(\bar{v}, \bar{w})
$$

and consequently

$$
\tilde{B}_{H}\left(v_{H}, w_{H}\right)-B_{H}\left(v_{H}, w_{H}\right)=\sum_{K, j} \omega_{K, j}\left(B_{K, j}(\bar{v}, \bar{w})-B_{K, j}\left(\bar{v}_{h}, \bar{w}_{h}\right)\right) .
$$

By (4.3) and (4.3), $\bar{v}$ can be written as

$$
\bar{v}=v_{H}^{*}(x)+\varepsilon\left(\frac{\varepsilon}{\tau}\right)^{q+2} e_{v}\left(\frac{x}{\varepsilon}\right) .
$$

where $e_{v}$ solves the periodic problem (4.3). Similarly, $\bar{v}_{h}$ is given by

$$
\bar{v}_{h}=v_{H, h}^{*}(x)+\varepsilon\left(\frac{\varepsilon}{\tau}\right)^{q+2} e_{v, h}\left(\frac{x}{\varepsilon}\right),
$$

where $v_{H, h}^{*}-v_{H, \text { lin }} \in S^{s}\left(I_{\delta}, \mathcal{T}_{h}\right)$ is an approximation of $v_{H}^{*}-v_{H, \text { lin }}$, and $e_{v, h}$ is an approximation of $e_{v}$. Hence,

$$
\begin{aligned}
\left|B_{K, j}(\bar{v}, \bar{w})-B_{K, j}\left(\bar{v}_{h}, \bar{w}_{h}\right)\right| \leq & \left|B_{K, j}\left(v_{H}^{*}, w_{H}^{*}\right)-B_{K, j}\left(v_{H, h}^{*}, w_{H, h}^{*}\right)\right| \\
& +\left(\frac{\varepsilon}{\tau}\right)^{q+2}\left|B_{K, j}\left(e_{v}, w_{H}^{*}\right)-B_{K, j}\left(e_{v, h}, w_{H, h}^{*}\right)\right|
\end{aligned}
$$




$$
\begin{aligned}
& +\left(\frac{\varepsilon}{\tau}\right)^{q+2}\left|B_{K, j}\left(v_{H}^{*}, e_{w}\right)-B_{K, j}\left(v_{H, h}^{*}, e_{w, h}\right)\right| \\
& +\left(\frac{\varepsilon}{\tau}\right)^{2 q+4}\left|B_{K, j}\left(e_{v}, e_{w}\right)-B_{K, j}\left(e_{v, h}, e_{w, h}\right)\right| .
\end{aligned}
$$

Since the first term is the leading order quantity in the micro error, we give an estimate of this term only. The higher order terms, bounded by $(\varepsilon / \tau)^{q+2}$, can be estimated similarly. We split the leading error term

$$
\left|B_{K, j}\left(v_{H}^{*}, w_{H}^{*}\right)-B_{K, j}\left(v_{H, h}^{*}, w_{H, h}^{*}\right)\right| \leq\left|B_{K, j}\left(v_{H}^{*}-v_{H, h}^{*}, w_{H}^{*}\right)\right|+\left|B_{K, j}\left(v_{H, h}^{*}, w_{H, h}^{*}-w_{H}^{*}\right)\right|
$$

using the standard triangular inequality and bound the first term on the right-hand side by,

$$
\begin{aligned}
\left|B_{K, j}\left(v_{H}^{*}-v_{H, h}^{*}, w_{H}^{*}\right)\right| & =\left|\int_{I_{\delta}} k_{\eta}(x) a^{\varepsilon}\left(x_{K, j}+x\right) \nabla\left(v_{H}^{*}-v_{H, h}^{*}\right) \cdot \nabla w_{H}^{*} d x\right| \\
& \leq C \eta^{-d}\left\|\nabla\left(v_{H}^{*}-v_{H, h}^{*}\right)\right\|_{L^{2}\left(I_{\delta}\right)}\left\|\nabla w_{H}^{*}\right\|_{L^{2}\left(I_{\delta}\right)} \\
& \leq C \eta^{-d} h^{s} \max _{i}\left\|\varepsilon \chi^{i}\left(\frac{\dot{\varepsilon}}{\varepsilon}\right)\right\|_{H^{s+1}\left(I_{\delta}\right)}\left|\nabla v_{H}\left(x_{K, j}\right)\right| \eta^{d / 2}\left|\nabla w_{H}\left(x_{K, j}\right)\right| \\
& \leq C\left(\frac{h}{\varepsilon}\right)^{s}\left|\nabla v_{H}\left(x_{K, j}\right)\right|\left|\nabla w_{H}\left(x_{K, j}\right)\right| .
\end{aligned}
$$

In the last line we used the fact that $\left|I_{\delta}\right|=O\left(\eta^{d}\right)$. The same bound holds for the second term because of the symmetry of $B_{K, j}$. As in the proof of the modeling error, we apply the Cauchy-Schwarz inequality and use the assumptions of the quadrature rule to obtain

$$
\sum_{K, j} \omega_{K, j}\left|B_{K, j}\left(v_{H}^{*}, w_{H}^{*}\right)-B_{K, j}\left(v_{H, h}^{*}, w_{H, h}^{*}\right)\right| \leq C\left(\frac{h}{\varepsilon}\right)^{s}\left\|v_{H}\right\|_{H^{1}(\Omega)}\left\|w_{H}\right\|_{H^{1}(\Omega)} .
$$

\section{Numerical results}

The goal of this section is twofold. On the one hand, we give numerical results corroborating the theory on the control of the modeling error. On the other hand, we show that FE-HMM with hyperbolic micro problems can be used even in situations beyond the scope of the convergence analysis above, e.g. in nonperiodic media. To demonstrate the flexibility and versatility of the method we consider an elliptic, a parabolic, and a hyperbolic example with Dirichlet, Neumann, or periodic boundary conditions. Furthermore, we alternate the macro and the micro solver, the quadrature formula, and the averaging kernel between the examples.

5.1. Modeling error. We provide numerical results showing that the modeling error can be arbitrarily reduced by taking smoother kernels. In the spirit of Theorem 4.7 we consider first the difference between the bilinear forms $B_{H}^{0}$ and $B_{H}$ in one and two dimensions. To this end let

$$
a^{\varepsilon}(x)=1.1+0.5 \sin \left(2 \pi \frac{x}{\varepsilon}\right)
$$

in one dimension and

$$
a^{\varepsilon}(x)=\left(1.1+0.5 \sin \left(2 \pi \frac{x_{1}}{\varepsilon}\right)\right)\left(1.1+0.5 \sin \left(2 \pi \frac{x_{2}}{\varepsilon}\right)\right) .
$$


in two dimensions. For both coefficients the exact homogenized coefficients can be computed analytically [21]. Since both bilinear forms consist of a sum over all quadrature point, we restrict our analysis to a single summand. Namely, we compute

$$
\left|B_{K, j}\left(\bar{v}_{h}, \bar{w}_{h}\right)-a^{0}\left(x_{K, j}\right) \nabla v_{H}\left(x_{K, j}\right) \cdot \nabla w_{H}\left(x_{K, j}\right)\right|,
$$

where $\bar{v}_{h}$ and $\bar{w}_{h}$, defined in (3.1), are the averages of micro solution constrained by $v_{H}$ and $w_{H}$, respectively.

We choose $x_{K, j}=0, v_{H}=w_{H}=x$ and fix the size of the averaging domain in space and time, while refining the small scale parameter $\varepsilon$. For the one-dimensional example shown on the left-hand side of Figure 5.1 we set $\eta=\tau=0.03$ and $h=\varepsilon / 40$. For the twodimensional example shown on right-hand side we set $\eta=\tau=0.01$ and $h=\varepsilon / 15$. The FE-HMM bilinear form is computed twice, once with a kernel $k \in \mathbb{K}^{3,5}$ and once with $k \in \mathbb{K}^{3,7}$. We recover the expected convergence rate $\mathcal{O}\left((\varepsilon / \eta)^{q+2}\right)$ experimentally (see Figure 5.1). The oscillations in the convergence curves can be explained as follows: If $\eta$ is an integer multiple of $\varepsilon$ the averaging is more accurate, but even if $\eta / \varepsilon$ is not an integer FE-HMM converges as $\varepsilon \rightarrow 0$.

Difference between homogenized and FE-HMM bilinear form

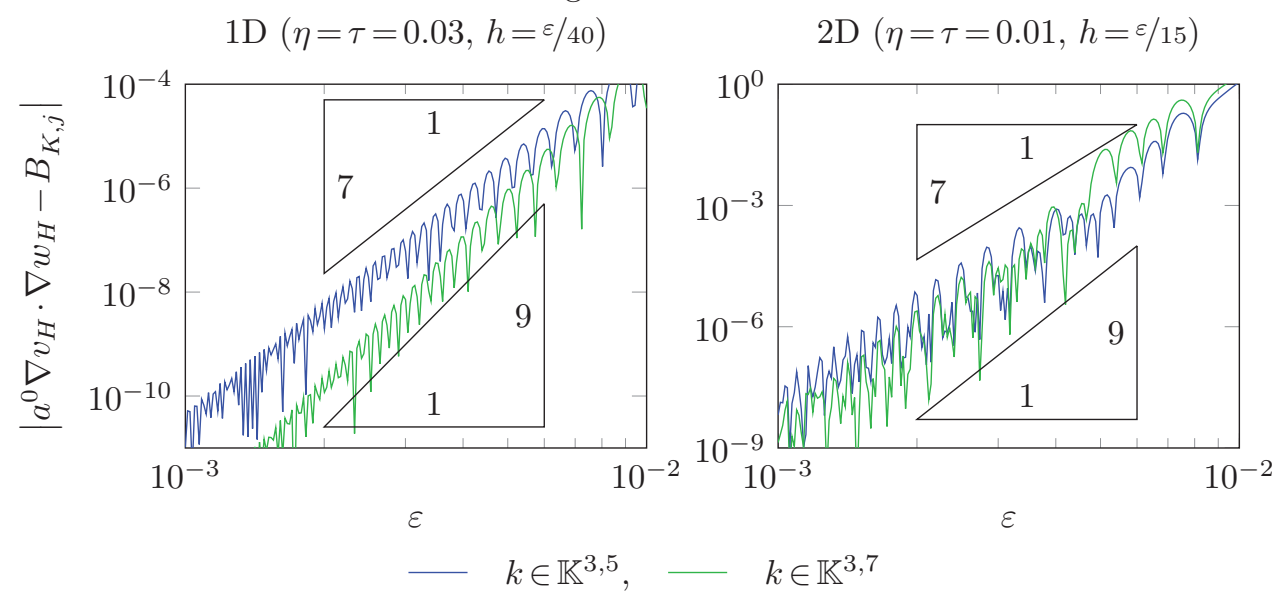

FIG. 5.1. Difference between the homogenized and the FE-HMM bilinear form for one (left) and two (right) dimensions. We fix $\eta$ and $\tau$ and let the small scale parameter $\varepsilon$ tend to zero. For a kernel $k \in \mathbb{K}^{3,5}$ we observe convergence of order 7 and for $k \in \mathbb{K}^{3,7}$ convergence of order 9. These results are in good agreement with the statement of Theorem 4.7.

To show that the results from the first experiment carry over to the actual modeling error we consider now the elliptic model problem $(2)$ in $\Omega=[0,1]$, where

$$
a^{\varepsilon}(x)=\sqrt{2}+\sin \left(2 \pi \frac{x}{\varepsilon}\right)
$$

with $\varepsilon=1 / 1000$ and $F(x)=\pi^{2} \sin (\pi x)$. Because our main interest is the modeling error we compute the difference between the FEM approximation $u_{H}^{0}$ of the homogenized equation and the FE-HMM solution $u_{H}$. Both solutions are computed with $\mathcal{P}^{1} \mathrm{FE}$ on the same uniform macro mesh with $H=1 / 64$. To reduce the micro error we solve the micro problems with high accuracy on a mesh with $h=\varepsilon / 1000$. Instead of diminishing $\varepsilon$ as in the previous example we increase $\eta$, while $\varepsilon$ remains fixed. In addition $\delta$ must be adapted as well. More precisely, we set $\tau=6 \varepsilon$ and

$$
\delta=\eta+\tau \sqrt{2.5} .
$$


This guarantees that no information from the boundary conditions interferes with the averaging procedure, since $a^{\varepsilon}(x)<2.5$ for all $x \in \Omega$. On the left-hand side of Figure 5.2 we show the $L^{2}$-difference between $u_{H}^{0}$ and $u_{H}$ computed with a kernel $k \in \mathbb{K}^{3,5}$. We observe a convergence rate of order 7 , as expected. The error we observe originates from the spatial approximation (FEM discretization of the micro problem and spatial averaging). In comparison the time averaging process results only in very small errors in this setting.

On the right-hand side of Figure 5.2 we show an excerpt of the same graph. Additionally, we display the modeling error of a standard FE-HMM scheme as described in [2]. There, elliptic cell problems are used and no averaging kernels are involved. Since $\delta$ is not an integer multiple of $\varepsilon$, we use a Dirichlet coupling condition. For the elliptic FE-HMM we used the same discretization parameters for the macro and the micro solver. Using elliptic micro problems the modeling error decays but very slowly compared to the modeling error obtained by using hyperbolic micro problems. One remarkable exception should be well noted: If the size of the sampling domain $I_{\delta}$ is an integer multiple of $\varepsilon$ the modeling error vanishes completely for periodic media [2, Proposition 14]. Because $\delta$ is given by (5.1) there is no case, where $\delta / \varepsilon$ is an integer. Nevertheless, we see clearly that the modeling error is minimal where this ratio equals almost 12 or 13 . Thus, we do not expect that in the second graph of Figure 5.2 the error of the elliptic FE-HMM vanishes. If $\delta / \varepsilon$ was an integer, we would still see the micro error emerging from the discretization of the micro problem. In this particular case it is advantageous to use elliptic micro problems. However, in more general situations, where the periodicity is not known, or in a non-periodic medium, the enhanced controllability of the modeling provided by the use of FE-HMM with hyperbolic micro problems might be worth while.

REMARK 5.1. Comparing our FE-HMM with hyperbolic micro problems with standard elliptic FE-HMM in terms of computational cost note first, that the macro solver is exactly the same for both cases. Hence, the same number of micro problems must be solved for both types of FE-HMM and since for both of them the micro problems are independent of each other a possible parallelization for the hyperbolic FE-HMM as straight forward as for standard FE-HMM. The difference is only due to solution of the micro problem. While for standard FE-HMM an elliptic problem must be solved for every quadrature node in the macro mesh, we need to solve hyperbolic problems. In addition slightly bigger sampling domains must be chosen, such that errors from inaccurate coupling conditions between macro and micro solution do not interfere. Note, that in standard FE-HMM there is no similar possibility to exclude this error source completely.

5.2. Elliptic problem. We consider the elliptic model problem (2) in $\Omega=[0,1]^{2}$ with homogeneous Dirichlet boundary conditions and a source given by

$$
F(x)=10 \exp \left(-10\left(\left(x_{1}-0.5\right)^{2}+\left(x_{2}-0.5\right)^{2}\right)\right) .
$$

The multiscale tensor is given by

$$
a^{\varepsilon}(x)=\left(\frac{41}{40}+\frac{9}{40} \sin \left(2 \pi \frac{x_{1}}{\varepsilon}\right)\right)\left(\frac{5}{4}+\frac{3}{4} \sin \left(2 \pi \frac{x_{1}}{\varepsilon}\right)\right),
$$

and we set $\varepsilon=1 / 100$. Because $a^{\varepsilon}$ is scalar, periodic and has separated variables $x_{1}$ and $x_{2}$, we can compute the homogenized tensor $a^{0}(x)=\operatorname{diag}(5 / 4,41 / 40)$, for all $x \in \Omega$ 


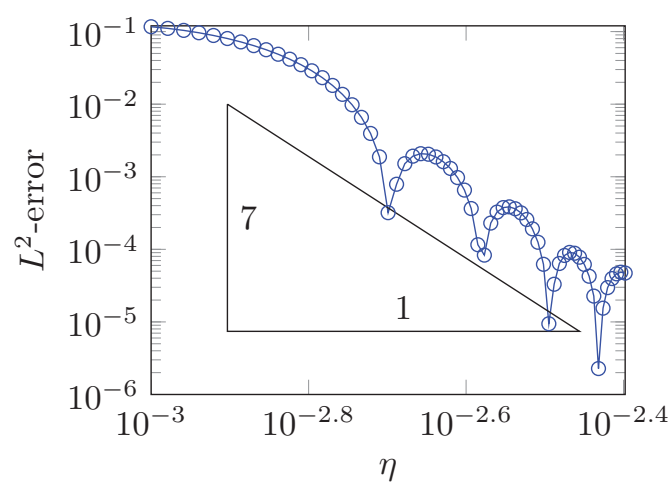

- hyperbolic micro problem,

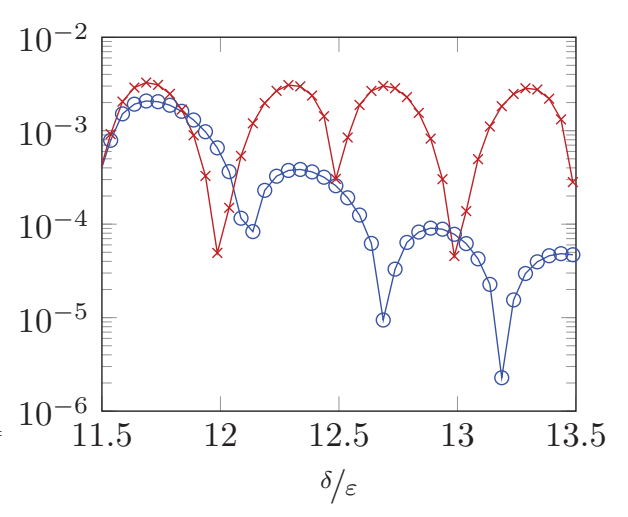

elliptic micro problem

FIG. 5.2. Difference between the FE-HMM solution $u_{H}$ and the discrete homogenized solution $u_{H}^{0}$ (left) and the comparison between the FE-HMM with elliptic and hyperbolic micro problems (right). We use a kernel $\kappa \in \mathbb{K}^{3,5}$, and for a fixed $\varepsilon$ we study the convergence as $\eta$ and $\tau$ grow. We observe convergence of order 7 (left plot). Moreover, the FE-HMM with hyperbolic micro problems leads to much smaller error, if the micro domain is large enough, in comparison to standard FE-HMM with elliptic micro problems (right).

(see [21]) Note, that even though $a^{\varepsilon}$ is scalar valued and thus isotropic for every $x \in \Omega$, the homogenized tensor is anisotropic.

We compare the FE-HMM solution $u_{H}$ with the discretized homogenized solution $u_{H}^{0}$ both computed with $\mathcal{Q}^{1} \mathrm{FE}$ on a uniform mesh with meshsize $H=0.1$ and a four point Gauss-Legendre quadrature formula given by the reference nodes and weights

$$
\hat{x}_{j}=\left(\frac{3 \pm \sqrt{3}}{6}, \frac{3 \pm \sqrt{3}}{6}\right) \quad \text { and } \quad \hat{\omega}_{j}=\frac{1}{4} \quad \text { for } j=1, \ldots, 4
$$

on the reference element $\hat{K}=[0,1]^{2}$. For the micro solver we use $\mathcal{Q}^{1} \mathrm{FE}$, too. In this example we would like to show again, that the FE-HMM with hyperbolic micro problems provides reliable solutions, even without knowing the exact length of the period. Therefore, we set $\eta=\tau=2.6 \varepsilon$ and the size of the sampling domain $\delta=6.711 \varepsilon$. Furthermore, we use a symmetric kernel $k \in \mathbb{K}^{3,9}$.

In Figure 5.3 we see that $u_{H}$ approximates well the homogenized solution, even though the small scale oscillations are not resolved with the coarse macro mesh. More precisely, the relative $H^{1}$-error between $u_{H}^{0}$ and $u_{H}$ is $8.28 \cdot 10^{-3}$.

5.3. Parabolic problem. Because we use FEM for the macro solver, the whole FE-HMM scheme can be as easily applied to complex geometry as standard FEMs. Here, we use the computational domain $\Omega$ depicted in Figure 5.4 and solve the heat equation (2) with no internal source, i.e. $F=0$. To model a uniformly distributed heat distribution at $t=0$, we set $f=0$. For $t>0$ we are heating from the left boundary $\Gamma_{1}^{D}$, while enforcing the original temperature at the right boundary $\Gamma_{2}^{D}$. Hence, we impose the following Dirichlet boundary condition:

$$
u(x, t)= \begin{cases}0, & \text { for } x \in \Gamma_{0}^{D} \text { and all } t>0 \\ 1, & \text { for } x \in \Gamma_{1}^{D} \text { and all } t>0\end{cases}
$$



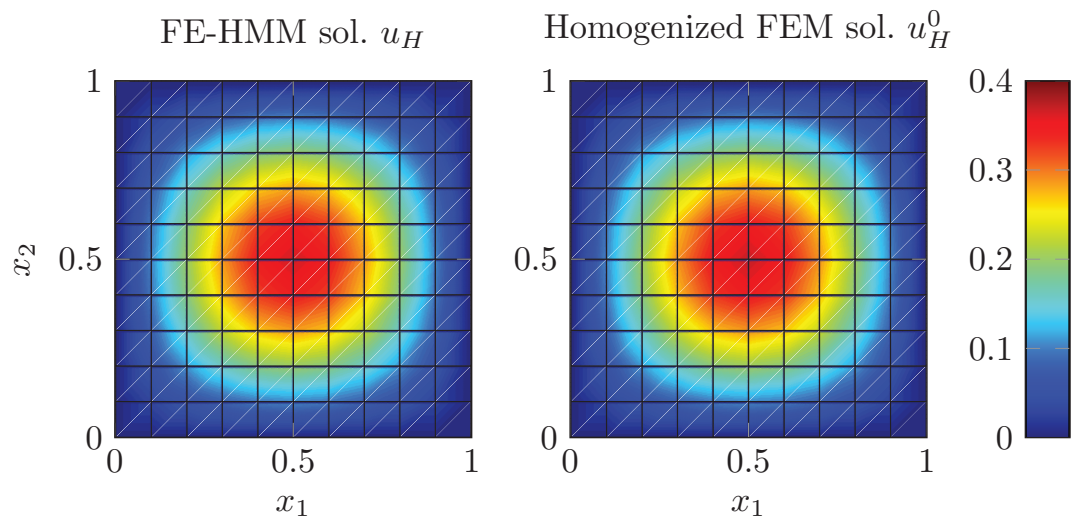

FIG. 5.3. Poisson equation: FE-HMM solution $u_{H}$ (right) and FEM solution of the homogenized equation with analytically computed tensor $a^{0}$ (left). The solutions coincide well, despite $\delta$, the size of the sampling domain, being not an integer multiple of $\varepsilon$.

We assume that the remaining boundary $\Gamma^{N}$ is perfectly insulated. Thus, we impose there a homogeneous Neumann boundary condition. The thermal diffusivity is modeled by

$$
a^{\varepsilon}(x)=\left(1.1+\frac{1}{2} \sin \left(2 \pi \frac{x_{1}}{\varepsilon}\right)\right)\left(1.1+\frac{1}{2}\left(\sin \left(2 \pi \frac{\sqrt{2} x_{2}}{\varepsilon}\right)+\sin \left(2 \pi \frac{x_{2}}{\varepsilon}\right)\right)\right)
$$

with $\varepsilon=1 / 40=0.025$. Note, that $a^{\varepsilon}$ is only $\varepsilon$-periodic in the $x_{1}$-direction, but not in $x_{2}$.

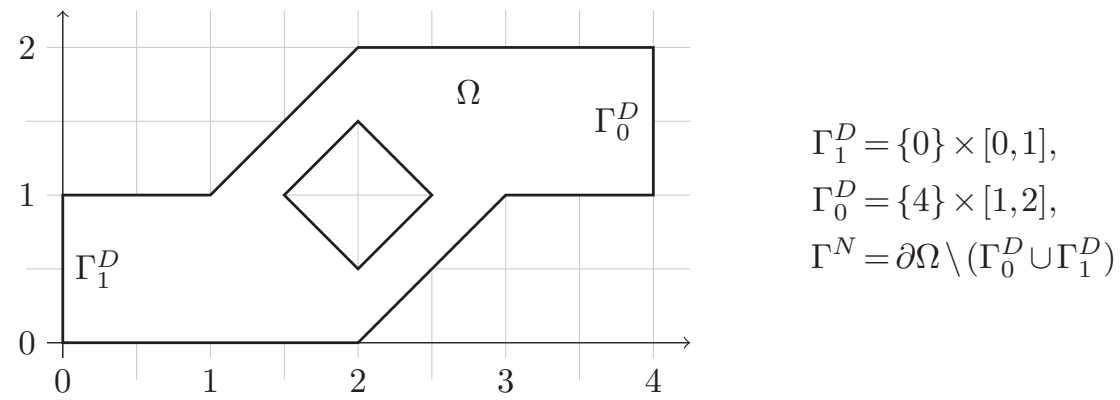

FIG. 5.4. Heat equation: Sketch of the computational domain $\Omega$. While imposing Dirichlet boundary conditions on $\Gamma_{0}^{D}$ and $\Gamma_{1}^{D}$, we impose a homogeneous Neumann condition on $\Gamma^{N}$.

Since $a^{0}$ can not be computed analytically in this case, we compare the FE-HMM solution with a direct numerical simulation (DNS), computed on a grid, that resolves all the oscillations of $a^{\varepsilon}$. To do so we used a triangular mesh with almost 700000 elements and piecewise linear FE. For the FE-HMM however we used for the macro solver $\mathcal{P}^{1} \mathrm{FE}$, too, but on a coarse mesh with only 138 elements. The other discretization parameters were chosen as follows: For linear finite elements it is enough to use the midpoint rule as quadrature formula. Hence, we only have to solve one micro problem per macroscopic element. We set the size of the sampling domain $\delta=3.59 \varepsilon$ and used $\mathcal{Q}^{1} \mathrm{FE}$ on a regular mesh with meshsize $h=\delta / 72$. For the averaging we used $k \in \mathbb{K}^{5,5}$ with $\eta=\tau=1.25 \varepsilon$. 
The spatial discretization of the heat equation, be it by FEM or FE-HMM, leads to system of first order ordinary differential equations that must be discretized as well. Here, we chose for the time-stepping the well known Crank-Nicolson scheme. Due to the stability of this implicit scheme, we could use the same timestep for the FE-HMM and the DNS solution. In Figure 5.5 we show three snapshots of the heating process. At $t=1.0$ a stable state has been reached. For a closer inspection this final state is shown again in Figure 5.6. The overall behavior of the FE-HMM and the DNS solution coincide well. However, the contour lines of the DNS solution display a microscopic wiggling behavior reflecting the multiscale structure of the underlying media. Since FE-HMM approximates the homogenized solution we do not recover these microscopic effects, as expected.
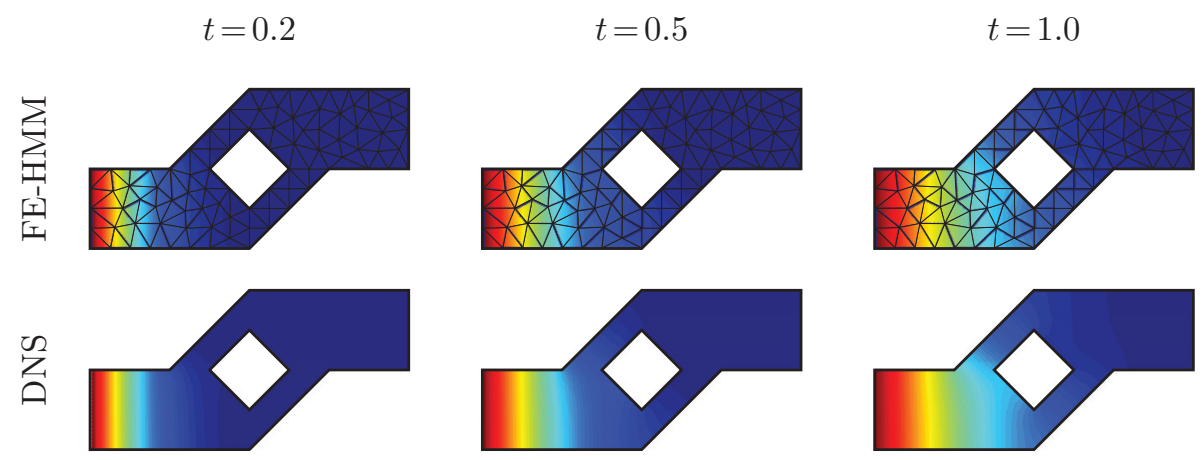

FIG. 5.5. Heat equation: Snapshots at $t=0.2, t=0.5$, and $t=1.0$ of the FE-HMM solution (top) and the DNS solution (bottom) show the close agreement of the FE-HMM with the true (DNS) solution. For the FE-HMM the mesh that we use is depicted as well. The mesh used for the DNS computation has over 5000 times more elements.

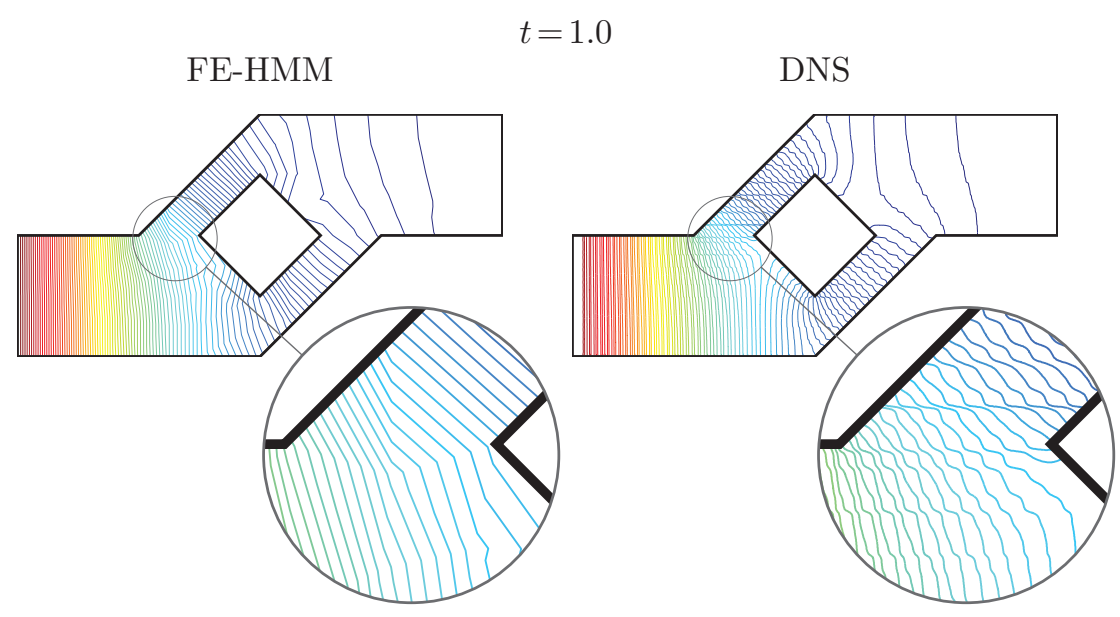

FIG. 5.6. Heat equation: Contour plot of the stable state reached at $t=1.0$ of the FE-HMM solution (left) and the DNS solution (right). The wiggles of the contour lines in the DNS solution, clearly observable on the zoom at $(1.25,1)$, are caused by the heterogeneities of the diffusivity. Since FE-HMM approximates the homogenized solution, these microscopic effects are not recovered. Yet, the overall behavior is well captured. 
5.4. Hyperbolic problem. For the final example we solve the one-dimensional wave equation (3.1) propagating through a complicated media given by

$$
a^{\varepsilon}(x)=1+\frac{1}{4}\left(\sin (\pi x)+\sin \left(\frac{2 \pi x}{\varepsilon(1-0.5 \cos (4 x))}\right),\right)
$$

with $\varepsilon=1 / 1000$. Not only is $a^{\varepsilon}$ highly oscillatory, but also the local average and the wave length of the oscillations vary over the computational domain $\Omega=[-1,1]$ (see Figure 5.7). As initial data we choose the Gaussian pulse $f(x)=\exp \left(-100 x^{2}\right)$ and $g(x)=0$ and set periodic boundary conditions.
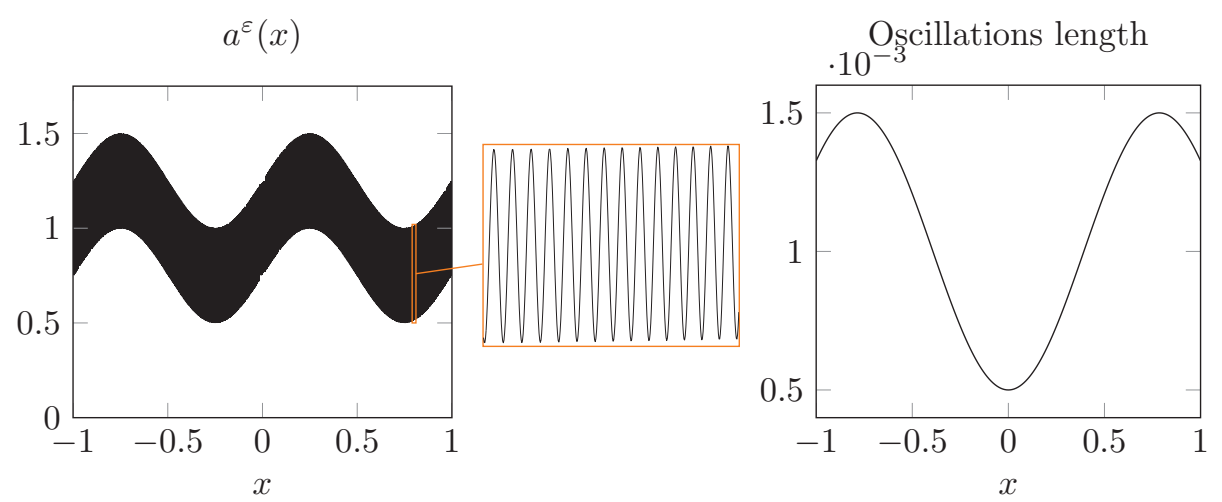

FIG. 5.7. Wave equation: Multiscale coefficient $a^{\varepsilon}(x)$ with a zoom at $x=0.8$ (left). Its oscillation length is not constant but varies (right).
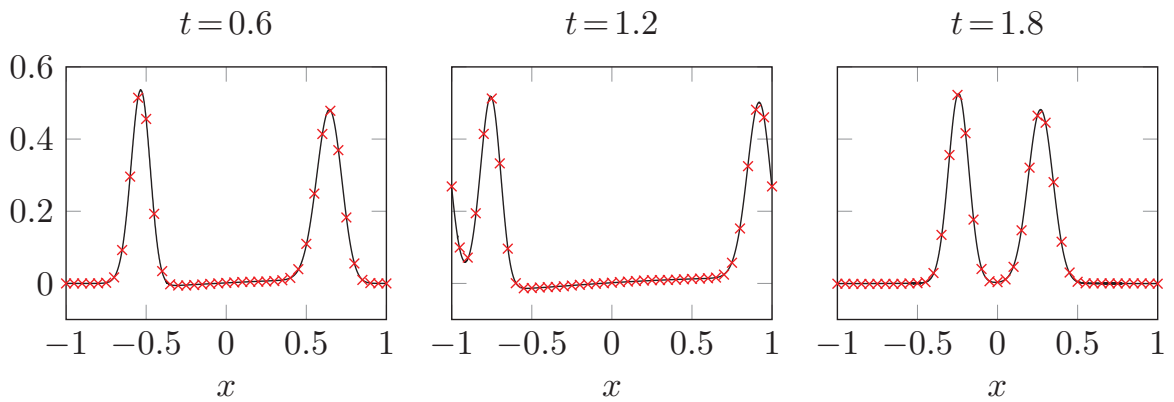

— Reference solution $u^{\varepsilon}(\mathrm{DNS}), \quad \times$ FE-HMM solution $u_{H}$

Fig. 5.8. Wave equation: Snapshots at $t=0.6, t=1.2$, and $t=1.8$ of the FE-HMM solution (red) and the DNS solution (black). Note that $u_{H}$ and the effective behavior of $u^{\varepsilon}$ coincide.

Similarly to the previous example for the heat equation, we compare the FE-HMM solution with a DNS computed on refined mesh. Here, however, we use cubic FE for both, the macro and the micro solver. For the spatial discretization we use a uniform mesh with macro meshsize $H=1 / 20$ and micro meshsize $h=10^{-4}$. For the timestepping we choose an explicit leap-frog scheme. Note that the CFL condition couples the timestep with the macro meshsize $H$. This means that larger timesteps are allowed compared with DNS discretization using standard FEM with a mesh that resolve all scales. This leads to an additional saving of computational costs. The FE-HMM 
parameters are chosen here as set as $\eta=\tau=10 \varepsilon$ and $\delta=22.25 \varepsilon$. Compared with the other examples $\eta, \tau$, and thus $\delta$ are chosen slightly bigger. This choice accounts for the variability of the oscillation length in the media.

In Figure 5.8 we show the reference solution $u^{\varepsilon}$ and the FE-HMM solution $u_{H}$ at three different times. Again, we observe that $u_{H}$ approximates well the effective behavior of $u^{\varepsilon}$. Once again, we would like to stress, that the spatial discretization of the FE-HMM scheme is exactly the same for all three examples above. Only the timestepping scheme must be adapted to the problem at hand.

\section{Conclusion}

We have proposed and analyzed a FE-HMM method for numerical approximation of homogenized solutions of linear multiscale PDEs. In particular, we consider elliptic, parabolic, and second order hyperbolic problems in heterogeneous media. The proposed method uses a second order hyperbolic PDE as the micro model, whereas the macro model is adjusted to the type of the underlying original problem. The modeling error of typical FE-HMM schemes are limited by an $O(\varepsilon / \eta)$ error. The FE-HMM developed here reduces the modeling error up to arbitrary rates in $\varepsilon / \eta$, without increasing the computational cost in terms of $\varepsilon$ in comparison to previous studies. Higher order rates are achieved by using smoother kernels while computing the bilinear form. Although, the present theory is restricted to the periodic media, numerical results confirm that the method performs well in one- and two-dimensional non-periodic media as well. Finally, we emphasize that the analysis for the parabolic and the hyperbolic problems are valid only for time independent coefficients and further studies are needed to treat the modeling error in the time dependent setting.

Acknowledgement. The research of Doghonay Arjmand is funded by Swedish eScience Research Center (SeRC). The financial support is gratefully acknowledged. The work of Christian Stohrer is partially supported by the ANR (Projet METAMATH) and the SNFS.

\section{REFERENCES}

[1] Assyr Abdulle, On a priori error analysis of fully discrete heterogeneous multiscale FEM, Multiscale Model. Simul., 4(2), 447-459, 2005.

[2] Assyr Abdulle, The finite element heterogeneous multiscale method: A computational strategy for multiscale PDEs, GAKUTO Internat. Ser. Math. Sci. Appl., 31, 133-181, 2009.

[3] Assyr Abdulle, Weinan E, Bjorn Engquist, and Eric Vanden-Eijnden, The heterogeneous multiscale method, Acta Numer., 21, 1-87, 2012.

[4] Assyr Abdulle and Marcus J. Grote, Finite element heterogeneous multiscale method for the wave equation, Multiscale Model. Simul., 9(2), 766-792, 2011.

[5] Assyr Abdulle, Marcus J. Grote, and Christian Stohrer, Finite element heterogeneous multiscale method for the wave equation: Long time effects, Multiscale Model. Simul., 12(3), 1230-1257, 2014.

[6] Assyr Abdulle and Gilles Vilmart, Coupling heterogeneous multiscale FEM with Runge-Kutta methods for parabolic homogenization problems: A fully discrete spacetime analysis, Math. Models Meth. Appl. Sci., 22(06), 1250002, 2012.

[7] Doghonay Arjmand, Analysis and Applications of the Heterogeneous Multiscale Methods for Elliptic and Hyperbolic Partial Differential Equation, Licentiate thesis, KTH, 2013.

[8] Doghonay Arjmand and Olof Runborg, Analysis of heterogeneous multiscale methods for long time wave propagation problems, Multiscale Model. Simul., 12(3), 1135-1166, 2014.

[9] Garth A. Baker and Vassilios A. Dougalis, The effect of quadrature errors on finite element approximations for second order hyperbolic equations, SIAM J. Numer. Anal., 13(4), 577598, 1976.

[10] Alain Bensoussan, Jaques-Louis Lions, and George Papanicolaou, Asymptotic Analysis for Periodic Structures, Stud. Math. Appl., North-Holland, 5, 1978. 
[11] Philippe G. Ciarlet, The Finite Element Method for Elliptic Problems, Classics in Applied Mathematics, SIAM, 40, 2002.

[12] Philippe G. Ciarlet and Pierre-Arnaud Raviart, The combined effect of curved boundaries ans numerical integration in isoparametric finite element methods, in The Mathematical Foundations of the Finite Element Method: With Application to Partial Differential Equations, Academic Press, 409-474, 1972.

[13] Doina Cioranescu and Patrizia Donato, An Introduction to Homogenization, Oxford Lecture Series in Mathematics and its Application, Oxford, University Press, 17, 1999.

[14] Weinan E, Principles of Multiscale Modeling, Cambridge University Press, 2011.

[15] Weinan E and Bjorn Engquist, The heterogeneous multiscale methods, Commun. Math. Sci., 1(1), 87-132, 2003.

[16] Weinan E, Bjorn Engquist, Xiantao Li, Weiqing Ren, and Erik Vanden-Eijnden, Heterogeneous multiscale methods: A review, Commun. Comput. Phys., 2(3), 367-450, 2007.

[17] Weinan E, Pingbing Ming, and Pingwen Zhang, Analysis of the heterogeneous multiscale method for elliptic homogenization problems, J. Amer. Math. Soc., 18(1), 121-156, 2005.

[18] Bjorn Engquist, Henrik Holst, and Olof Runborg, Multiscale methods for the wave equation, Proc. Appl. Math. Mech., 7(1), 1140903-1140904, 2007.

[19] Bjorn Engquist, Henrik Holst, and Olof Runborg, Multi-scale methods for wave propagation in heterogeneous media, Commun. Math. Sci., 9(1), 33-56, 2011.

[20] Bjorn Engquist and Yen-Hsi Tsai, Heterogeneous multiscale methods for stiff ordinary differential equations, Math. Comp., 74(252), 1707-1742, 2005.

[21] Vasilii V. Jikov, Serguei M. Kozlov, and Olga A. Oleinik, Homogenization of Differential Operators and Integral Functionals, Springer-Verlag, 1994.

[22] Mark G. Krein and Mark A. Rutman, Linear Operators that Leave Invariant a Cone in a Banach Space, Uspehi Matem. Nauk (N. S.) 3, 1(23), 3-95, 1948.

[23] Pingbing Ming and Pingwen Zhang, Analysis of the heterogeneous multiscale method for parabolic homogenization problems, Math. Comp., 76(257), 153-177, 2007.

[24] Pierre-Arnaud Raviart, The use of numerical integration in finite element methods for solving parabolic equations, in Topics in Numerical Analysis (Proc. Roy. Irish Acad. Conf., University Coll., Dublin, 1972), Academic Press, London, 233-264, 1973.

[25] Christian Stohrer, Finite Element Heterogeneous Multiscale Methods for the Wave Equation, PhD thesis, University of Basel, 2013.

[26] Xingye Yue and Weinan E, The local microscale problem in the multiscale modeling of strongly heterogeneous media: Effects of boundary conditions and cell size, J. Comput. Phys., 222(2), 556-572, 2007. 